\title{
Spectral Extension of the Quantum Group Cotangent Bundle
}

\author{
Alexei P. Isaev ${ }^{1}$, Pavel Pyatov ${ }^{1,2,3}$ \\ 1 Bogoliubov Laboratory of Theoretical Physics, Joint Institute for Nuclear Research, \\ 141980 Dubna, Moscow Region, Russia. \\ E-mail: isaevap@theor.jinr.ru; pyatov@theor.jinr.ru \\ 2 Max Planck Institute for Mathematics, Vivatsgasse 7, D-53111 Bonn, Germany \\ 3 Department of Mathematics, Higher School of Economics, Myasnitskaya 20, Moscow, Russia
}

Received: 11 June 2008 / Accepted: 24 November 2008

Published online: 28 March 2009 - (C) Springer-Verlag 2009

\begin{abstract}
The structure of a cotangent bundle is investigated for quantum linear groups $G L_{q}(n)$ and $S L_{q}(n)$. Using a $q$-version of the Cayley-Hamilton theorem we construct an extension of the algebra of differential operators on $S L_{q}(n)$ (otherwise called the Heisenberg double) by spectral values of the matrix of right invariant vector fields. We consider two applications for the spectral extension. First, we describe the extended Heisenberg double in terms of a new set of generators - the Weyl partners of the spectral variables. Calculating defining relations in terms of these generators allows us to derive $S L_{q}(n)$ type dynamical R-matrices in a surprisingly simple way. Second, we calculate an evolution operator for the model of the $q$-deformed isotropic top introduced by A.Alekseev and L.Faddeev. The evolution operator is not uniquely defined and we present two possible expressions for it. The first one is a Riemann theta function in the spectral variables. The second one is an almost free motion evolution operator in terms of logarithms of the spectral variables. The relation between the two operators is given by a modular functional equation for the Riemann theta function.
\end{abstract}

\section{Introduction}

A notion of a Heisenberg double over the quantum group has been formulated and attracted substantial researcher's interest in the early 90-s [AF.91, S, SWZ.92, SWZ.93]. From the algebraic point of view it is a smash product algebra (see [M]) of the quantum group (or, the quantized universal enveloping algebra) and its dual Hopf algebra (see [D.86,FRT]). In the differential geometric interpretation it may be viewed as an algebra of quantized differential operators over a group or, equivalently, as an algebra of quantized functions over a cotangent bundle of the group. Since the group's cotangent bundle serves a typical phase space for integrable classical dynamics, it is natural to attach the same role to the Heisenberg double over the quantum group for quantum physical models. As a test example, a model of the $q$-deformed isotropic top was suggested in [AF.91, AF.92]. A discrete time evolution in this model is given by a series of automor- 
phisms of the Heisenberg double. It turns out however that finding an explicit expression for the model's evolution operator is not just a technical problem. ${ }^{1}$ The automorphisms defining the evolution by no means can be treated as inner ones in the original algebra and so, for a proper realization of the $q$-top one needs an appropriate extension of the Heisenberg double.

Also stimulated by the invention of quantum groups were general studies of the algebras whose generators satisfy quadratic relations (see [PP] and references therein) and investigations of minor identities for matrices over noncommutative rings [GR.91, GR.92, KL]. These two lines of research are meeting together in the theory of the socalled quantum matrix algebras [H,IOP.99] whose structure theory can be developed in a full analogy with the usual matrix analysis. In particular, one can define quantum versions of the matrix trace and determinant [FRT], introduce notions of a spectrum and a power of the quantum matrix, and formulate the Cayley-Hamilton theorem (see [GPS.97,IOP.99, OP.05] and references therein).

A remarkable fact about quantum matrix algebras is that their most known examples — the RTT algebra [FRT] and the reflection equation algebra [KS] — serve as the building blocks for a construction of the quantum group differential geometry in general [SWZ.92] and so, also for the Heisenberg double. It is the aim of the present paper to apply the structure results on the quantum matrix algebras for investigation of the dynamics of the isotropic $q$-top. Following [AF.91,S,SWZ.92] we begin with a definition of the Heisenberg double as a smash product algebra of a pair of quantum matrix algebras. These are the RTT algebra, playing the role of quantized functions over a group, and the reflection equation algebra, interpreted as quantized right invariant differential operators over a group. We then consider a central extension of the reflection equation algebra by the spectrum of it's generating matrix of quantized right invariant vector fields, and define a proper (non-central) extension of the whole Heisenberg double by these spectral variables. Finally, after the spectral extension is made, the evolution of the isotropic $q$-top becomes an inner automorphism of the Heisenberg double. ${ }^{2}$ Constructing the evolution operator is then straightforward.

The paper is organized as follows. In the next section we recall some facts about the universal R-matrix and the R-matrix techniques. We are mainly discussing the case of (numeric) R-matrices of type $G L_{q}(n)$. These type R-matrices are later on used for description of the cotangent bundles (or, the Heisenberg doubles) over quantum linear groups.

In Sect. 3 we introduce the RTT algebra, the reflection equation algebra and define their smash product algebra - the Heisenberg double. We are describing the algebras of the two linear types $-G L_{q}(n)$ and $S L_{q}(n)$. For the reflection equation algebra we formulate in these cases the Cayley-Hamilton theorem and use it for the spectral extension of the Heisenberg double. This is the first main result of the paper (see theorem 3.27).

The Heisenberg double is initially defined in terms of the quantized right invariant vector fields. In order to demonstrate the left-right symmetry of the Heisenberg double, in subsection 3.4 we describe it using quantized left invariant vector fields. We also derive explicit relations between the spectra of the matrices of left and right generators, see corollaries 3.17 and 3.34. To keep clearness of a presentation some technical lemmas are moved from this subsection to appendix B.

\footnotetext{
1 This problem was suggested to the authors by L.D. Faddeev in summer of 1996, during the Alushta conference "Nonlocal, nonrenormalizable field theories".

2 Strictly speaking, one has to extend the algebra by a formal power series in the spectral variables.
} 
The spectral extension suggests yet another distinguished generating set for the Heisenberg double, namely, the one which satisfy the simplest possible - Weyl algebraic relations with the spectral variables. In the subsection 3.5 we derive defining relations for this set, see theorem 3.36. Quite expectantly, the relations involve dynamical R-matrices whose dynamical arguments are the spectral variables (see corollary 3.37). Surprising facts are that the dynamical R-matrices are coming in pairs, and that they are derived by solving a simple system of (at most three) linear equations.

Section 4 is devoted to solving a dynamical problem for the isotropic $q$-top. This is our second main result. Noticing that an evolution operator of the model is not uniquely defined, we derive two different expressions for it. The first one is given in terms of the Riemann theta function whose matrix of periods is proportional to Gram matrix of the lattice $A_{n-1}^{*}$, see relations (4.3.4), (4.3.5). This solution converges for $|q|<1$, or for $q$ a rational root of 1 . The second solution converging for arbitrary values of $q$ is given in terms of logarithms of the spectral variables, see (4.4.2), (4.4.3). The idea for the logarithmic substitution (that means passing from Weyl type to Heisenberg type commutation relations) was suggested to authors by L.D. Faddeev (for argumentation see [F.94,F.95]). The evolution in the logarithmic variables reduces to an almost free motion. A relation between the two solutions is given then by a modular functional equation for Riemann theta function (4.4.4).

Concluding the introduction we would like to mention a number of open problems which, in our opinion, deserve further investigation.

First of all, it is straightforward to formulate a problem of spectral extension for the Heisenberg doubles over orthogonal and symplectic quantum groups and over quantum linear supergroups. Technical prerequisites for this were developed, respectively, in [OP.05] and [GPS.05,GPS.06]. As well, it would be interesting to construct spectral extensions for the cases of real quantum groups. We believe that a correct setting for this problem is suggested in [AF.92].

Another interesting problem is an extension of a modular double construction [F.95, F.99] (see also [KLS,GKL]) for the case of Heisenberg double over quantum group. A starting point for investigation here would be a modular functional relation (4.4.4) between the two evolution operators constructed in section 4 . Riemann theta function standing in the denominator in this relation could be considered as an evolution operator for the modular dual Heisenberg double.

At last, an observation that a ribbon element serves a $q$-top evolution operator on the smash product algebra of a ribbon Hopf algebra with it's dual Hopf algebra (see example 4.3) could open a way for the spectral extension of a quasi-triangular Hopf algebra. A partial step in this direction is made in appendix A, where pairing of the quasi-triangular Hopf algebra with its dual Hopf algebra is extended for the set of spectral variables, see corollary A.2.

\section{R-Matrices}

In this introductory section we collect some necessary information about R-matrices and an R-matrix technique.

2.1. Universal R-matrix. First, we recall a few basic notions from the theory of quasitriangular Hopf algebras [D.86,D.89] and ribbon Hopf algebras [RT] (for review see $[\mathrm{ChP}, \mathrm{KSch}])$. 
Let $\mathfrak{A}$ be a Hopf $\mathbb{C}$-algebra supplied with a unit $1: \mathbb{C} \rightarrow \mathfrak{A}$, a counit $\epsilon: \mathfrak{A} \rightarrow \mathbb{C}$, a product $\mathbf{m}: \mathfrak{A} \otimes \mathfrak{A} \rightarrow \mathfrak{A}$, a coproduct $\Delta: \mathfrak{A} \rightarrow \mathfrak{A} \otimes \mathfrak{A}$, and an antipode $S: \mathfrak{A} \rightarrow \mathfrak{A}$ mappings subject to standard axioms.

A Hopf algebra $\mathfrak{A}$ is called almost cocommutative if there exists an invertible element $\mathcal{R} \in \mathfrak{A} \otimes \mathfrak{A}$ that intertwines the coproduct $\Delta$ and the opposite coproduct $\Delta^{o p}$ (in Sweedler's notation: $\Delta^{o p}(x)=x_{(2)} \otimes x_{(1)}$ if $\left.\Delta(x)=x_{(1)} \otimes x_{(2)}\right)$

$$
\mathcal{R} \Delta(x)=\Delta^{o p}(x) \mathcal{R} \quad \forall x \in \mathfrak{A}_{\mathcal{R}}
$$

In this case the element $\mathcal{R}$ is called a universal $R$-matrix, and the corresponding almost cocommutative Hopf algebra is denoted as $\mathfrak{A}_{\mathcal{R}}$. The algebra $\mathfrak{A}_{\mathcal{R}}$ is called quasi-triangular if additionally $\mathcal{R}$ satisfies relations

$$
(\Delta \otimes i d) \mathcal{R}=\mathcal{R}_{13} \mathcal{R}_{23}, \quad(\text { id } \otimes \Delta) \mathcal{R}=\mathcal{R}_{13} \mathcal{R}_{12},
$$

where $\mathcal{R}_{12}=\mathcal{R} \otimes 1, \mathcal{R}_{23}=1 \otimes \mathcal{R}$, and $\mathcal{R}_{13}=\sum_{i} a_{i} \otimes 1 \otimes b_{i}$ for $\mathcal{R}=\sum_{i} a_{i} \otimes b_{i}$. Relations (2.1.1), (2.1.2) together imply an equality

$$
\mathcal{R}_{12} \mathcal{R}_{13} \mathcal{R}_{23}=\mathcal{R}_{23} \mathcal{R}_{13} \mathcal{R}_{12}
$$

which is called the Yang-Baxter equation.

In the almost cocommutative case an element $u:=\mathbf{m}(S \otimes i d)\left(\mathcal{R}_{21}\right) \in \mathfrak{A}_{\mathcal{R}}$ is invertible. In terms of $u$ the square of the antipode is expressed as

$$
S^{2}(x)=u x u^{-1} \quad \forall x \in \mathfrak{A}_{\mathcal{R}} .
$$

In the quasi-triangular case one has following formulas:

$$
\begin{array}{ll}
S(u)=\mathbf{m}(i d \otimes S)\left(\mathcal{R}_{12}\right), & u^{-1}=\mathbf{m}\left(i d \otimes S^{2}\right)\left(\mathcal{R}_{21}\right), \\
\Delta(u)=\left(\mathcal{R}_{21} \mathcal{R}_{12}\right)^{-1} u \otimes u, & \mathcal{R}(u \otimes u)=(u \otimes u) \mathcal{R} .
\end{array}
$$

An element $u S(u)=S(u) u$ (in the almost cocommutative case) belongs to the center of $\mathfrak{A}_{\mathcal{R}}$. A central extension of the quasi-triangular Hopf algebra $\mathfrak{A}_{\mathcal{R}}$ by a so-called ribbon element $v$ such that

$$
v^{2}=u S(u), \quad \Delta(v)=\left(\mathcal{R}_{21} \mathcal{R}_{12}\right)^{-1} v \otimes v
$$

is called a ribbon Hopf algebra. The ribbon element also fulfills relations

$$
\epsilon(v)=1, \quad S(v)=v, \quad \mathcal{R}(v \otimes v)=(v \otimes v) \mathcal{R} .
$$

Throughout this paper our basic reference example of the quasi-triangular Hopf algebra $\mathfrak{A}_{\mathcal{R}}$ is the quantized universal enveloping algebra $U_{q}(\mathfrak{g})$ of a complex Lie algebra $\mathfrak{g}=\mathfrak{s l}(n)$ [D.86,J.85,J.86]. 
2.2. Braid groups and their $R$-matrix representations. In the rest of the section we introduce standard notation and recall basic results on R-matrix representations of the braid groups.

The braid group $\mathcal{B}_{k}$ in Artin's presentation is given by a set of generators $\left\{\sigma_{i}\right\}_{i=1}^{k-1}$ and relations

$$
\begin{array}{ll}
\sigma_{i} \sigma_{i+1} \sigma_{i}=\sigma_{i+1} \sigma_{i} \sigma_{i+1} & \forall i=1,2, \ldots, k-1, \\
\sigma_{i} \sigma_{j}=\sigma_{j} \sigma_{i} & \forall i, j:|i-j|>1 .
\end{array}
$$

Let $V$ be a finite dimensional $\mathbb{C}$-linear space. For any operator $X \in \operatorname{End}\left(V^{\otimes 2}\right)$ and for all integer $i>0, j>0$ denote

$$
X_{i}:=I^{\otimes(i-1)} \otimes X \otimes I^{\otimes(j-1)} \in \operatorname{End}\left(V^{\otimes(i+j)}\right),
$$

where $I \in \operatorname{Aut}(V)$ is the identity operator. ${ }^{3}$ We also use notation $X_{i j}$ for an operator in End $\left(V^{\otimes k}\right), 1 \leq i \neq j \leq k$, acting as $X$ in component spaces $V$ with labels $i$ and $j$ and as identity in the rest. In these notations $X_{i+1} \equiv X_{i}$.

An operator $R \in \operatorname{Aut}(V \otimes V)$ satisfying equality

$$
R_{1} R_{2} R_{1}=R_{2} R_{1} R_{2} \text {, }
$$

is called an R-matrix. Any R-matrix generates representations $\rho_{R}$ of the braid groups $\mathcal{B}_{k}$, $k=2,3, \ldots$,

$$
\rho_{R}: \quad \mathcal{B}_{k} \rightarrow \operatorname{Aut}\left(V^{\otimes k}\right), \quad \rho_{R}\left(\sigma_{i}\right)=R_{i}, \quad 1 \leq i \leq k-1 .
$$

By a slight abuse of notation we assign the same symbol $\rho_{R}$ to the R-matrix representations of the braid groups $\mathcal{B}_{k}$ for different values of index $k$. This should not cause problems as the braid groups admit a series of monomorphisms commuting with $\rho_{R}$,

$$
\mathcal{B}_{k} \hookrightarrow \mathcal{B}_{k+1}: \quad \sigma_{i} \mapsto \sigma_{i} \quad \forall i=1, \ldots k-1 .
$$

Definition 2.1. An R-matrix $R$ is called skew invertible if there exists an operator $\Psi_{R} \in \operatorname{End}\left(V^{\otimes 2}\right)$ such that

$$
\operatorname{Tr}_{(2)} R_{12} \Psi_{R 23}=\operatorname{Tr}_{(2)} \Psi_{R 12} R_{23}=P_{13} .
$$

Here by $\operatorname{Tr}_{(i)}$ we denote trace operation in $i^{\text {th }}$ space, and by $P$ - the permutation operator: $P(u \otimes v)=v \otimes u \forall u, v \in V$.

With any skew invertible R-matrix $R$ we associate a pair of operators $D_{R}, C_{R} \in \operatorname{End}(V)$

$$
D_{R 1}=\operatorname{Tr}_{(2)} \Psi_{R 12}, \quad C_{R 2}=\operatorname{Tr}_{(1)} \Psi_{R 12},
$$

which, by (2.2.6), satisfy equalities

$$
\operatorname{Tr}_{(2)} R_{12} D_{R 2}=I_{1}, \quad \operatorname{Tr}_{(1)} C_{R 1} R_{12}=I_{2} .
$$

Further properties of the operators $D_{R}$ and $C_{R}$ are summarized below.

\footnotetext{
3 Strictly speaking a proper notation for the 1.h.s. of (2.2.3) would be, say, $X_{i}^{(i+j)}$. We use the shortened notation $X_{i}$ since a dependence on $j$ is not critical for our considerations. All formulas below make sense if the index $j$ is large enough. A minimal possible value for $j$ in each case is obvious from the context.
} 
Proposition 2.2 [Is.04, O]. Let $R$ be a skew-invertible R-matrix. The operators $D_{R}$ and $C_{R}(2.2 .7)$ satisfy equalities

$$
\begin{array}{ll}
D_{R 1} I_{2}=\operatorname{Tr}_{(3)} D_{R 3} R_{2}^{ \pm 1} P_{12} R_{2}^{\mp 1}, & C_{R 3} I_{2}=\operatorname{Tr}_{(1)} C_{R 1} R_{1}^{ \pm 1} P_{23} R_{1}^{\mp 1}, \\
R_{12} D_{R 1} D_{R 2}=D_{R 1} D_{R 2} R_{12}, & R_{12} C_{R 1} C_{R 2}=C_{R 1} C_{R 2} R_{12} .
\end{array}
$$

Let $W$ be a $\mathbb{C}$-linear space. For any skew invertible R-matrix $R$ we define an R-trace $\operatorname{map}^{4} \operatorname{Tr}_{R}: \operatorname{End}_{W}(V) \rightarrow W$,

$$
Y \mapsto \operatorname{Tr}_{R}(Y):=\operatorname{Tr}\left(D_{R} Y\right), \quad Y \in \operatorname{End}_{W}(V) .
$$

Following properties of the R-trace are simple consequences of the relations given in Proposition 2.2.

Corollary 2.3. Let $R$ be a skew invertible R-matrix. For any operator $Y \in \operatorname{End}_{W}(V)$ the $\mathrm{R}$-trace associated with $R$ satisfies relations

$$
\operatorname{Tr}_{R}(2)\left(R_{12}^{\varepsilon} Y_{1} R_{12}^{-\varepsilon}\right)=I_{1} \operatorname{Tr}_{R}(Y),
$$

where $\varepsilon= \pm 1$ and the symbol $\operatorname{Tr}_{R}(i)$ denotes taking the $\mathrm{R}$-trace in $i^{t h}$ space.

For an element $x^{(k)} \in \mathbb{C}\left[\mathcal{B}_{k}\right]$ denote $X_{R}^{(k)}:=\rho_{R}\left(x^{(k)}\right) \in \operatorname{End}\left(V^{\otimes k}\right)$. The following cyclic property

$$
\operatorname{Tr}_{R}(1, \ldots, k)\left(X_{R}^{(k)} Y^{(k)}\right)=\operatorname{Tr}_{R}(1, \ldots, k)\left(Y^{(k)} X_{R}^{(k)}\right)
$$

is fulfilled for any $k \geq 1$ and $Y^{(k)} \in \operatorname{End}_{W}\left(V^{\otimes k}\right)$, and for all $x^{(k)} \in \mathbb{C}\left[\mathcal{B}_{k}\right]$.

Example 2.4. Permutation $P: P(u \otimes v):=v \otimes u \forall u, v \in V$, is the skew invertible $\mathrm{R}$-matrix. The Identity operator $I^{\otimes 2}$ is the R-matrix which is not skew invertible.

Example 2.5. Assume that the quasi-triangular Hopf algebra $\mathfrak{A}_{\mathcal{R}}$ admits a representation

$$
\rho_{V}: \mathfrak{A}_{\mathcal{R}} \rightarrow \operatorname{End}(V) .
$$

As follows from the Yang-Baxter equation (2.1.3) an operator

$$
R:=\eta P\left(\rho_{V} \otimes \rho_{V}\right)(\mathcal{R}),
$$

satisfies relation (2.2.4). Here the scaling factor $\eta \in\{\mathbb{C} \backslash 0\}$ is introduced for the sake of future convenience.

The R-matrix (2.2.11) is skew invertible, its skew inverse matrix is given by formula (see, e.g., [O], Sect. 4.1.2)

$$
\Psi_{R}=\eta^{-1} P\left(\rho_{V} \otimes \rho_{V}\right)((i d \otimes S) \mathcal{R}) .
$$

The matrices $D_{R}$ and $C_{R}$ associated with the R-matrix (2.2.11) are:

$$
D_{R}=\eta^{-1} \rho_{V}(u), \quad C_{R}=\eta^{-1} \rho_{V}(S(u)) .
$$

Both, they are invertible and their properties (2.2.9) are descending from (2.1.5).

\footnotetext{
4 This map is often called a quantum trace or, shortly, a $q$-trace. In our opinion, the name R-trace is more appropriate to it.
} 
2.3. Hecke algebras and Hecke type R-matrix. An A-type Hecke algebra $\mathcal{H}_{k}(q)$ is a quotient algebra of the group algebra $\mathbb{C}\left[\mathcal{B}_{k}\right](2.2 .1),(2.2 .2)$ by relations

$$
\left(\sigma_{i}-q 1\right)\left(\sigma_{i}+q^{-1} 1\right)=0 \quad \forall 1 \leq i \leq k-1 .
$$

Under the following conditions on the parameter $q$ :

$$
\text { [k] } i_{q}:=\left(q^{i}-q^{-i}\right) /\left(q-q^{-1}\right) \neq 0 \quad \forall i=2,3, \ldots, k,
$$

the algebra $\mathcal{H}_{k}(q)$ is isomorphic to the group algebra of the symmetric group $\mathbb{C}\left[S_{k}\right]$ and, hence, semisimple. It's irreducible representations as well as its central idempotents are labeled by a set of partitions $\lambda \vdash k$. We are particularly interested in a series of idempotents corresponding to the one dimensional representations $\lambda=\left(1^{k}\right), k=1,2, \ldots$. These idempotents - we denote them as $a^{(k)}-$ admit a recursive construction (see, e.g., [HIOPT], Sect. 1, or [GPS.97], Sect. 2.3, or [TW], Lemma 7.2)

$$
\begin{aligned}
& a^{(1)}=1, \quad a^{(k)}=\frac{(k-1)_{q}}{k_{q}} a^{(k-1)}\left(\frac{q^{k-1}}{(k-1)_{q}} 1-\sigma_{k-1}\right) a^{(k-1)} \\
& =\frac{(k-1)_{q}}{k_{q}} a^{(k-1) \uparrow 1}\left(\frac{q^{k-1}}{(k-1)_{q}} 1-\sigma_{1}\right) a^{(k-1) \uparrow 1} \quad \forall k=2,3, \ldots,
\end{aligned}
$$

where we use the symbol $x^{(k) \uparrow 1} \in \mathcal{H}_{k+1}(q)$ for an image of the element $x^{(k)} \in \mathcal{H}_{k}(q)$ under the following algebra monomorphism (cf. with (2.2.5)):

$$
\mathcal{H}_{k} \hookrightarrow \mathcal{H}_{k+1}: \quad \sigma_{i} \mapsto \sigma_{i+1} \quad \forall i=1, \ldots k-1 .
$$

The idempotents $a^{(k)}$ obey relations

$$
\begin{array}{ll}
a^{(k)} \sigma_{i}=\sigma_{i} a^{(k)}=-q^{-1} a^{(k)} & \forall i=1,2, \ldots, k-1, \\
a^{(k)} a^{(i) \uparrow j}=a^{(i) \uparrow j} a^{(k)}=a^{(k)}, & \text { if } i+j \leq k .
\end{array}
$$

An R-matrix $R$ satisfying the quadratic minimal characteristic identity is called a Hecke type R-matrix. By an appropriate rescaling of $R$ one always can turn its characteristic identity to a form

$$
(R-q I)\left(R+q^{-1} I\right)=0 .
$$

In this case the corresponding representations $\rho_{R}$ become representations of the Hecke algebras $\mathcal{H}_{k}(q)$,

$$
\rho_{R}: \quad \mathcal{H}_{k}(q) \rightarrow \operatorname{Aut}\left(V^{\otimes k}\right), \quad \rho_{R}\left(\sigma_{i}\right)=R_{i}, \quad 1 \leq i \leq k-1 .
$$

We reserve a special notation for the R-matrix images of idempotents $a^{(k)}$ :

$$
A^{(k)}:=\rho_{R}\left(a^{(k)}\right), \quad A^{(k) \uparrow 1}:=\rho_{R}\left(a^{(k) \uparrow 1}\right) \quad \forall k \geq 1 .
$$

We also put $A^{(0)}:=1$. The elements $A^{(k)}$ will be further referred to as $k$-antisymmetrizers.

Remark 2.6. The R-matrix analogues of relations (2.3.2)-(2.3.5) have been described in the literature (see $[\mathrm{J} .86, \mathrm{G}]$ ) even earlier than their algebraic prototypes. 
2.4. $G L_{q}(n)$ type R-matrix.

Definition 2.7. Consider a Hecke type R-matrix R. Assume that the parameter $q$ in its characteristic identity (2.3.6) satisfies conditions [n] (2.3.1), so that antisymmetrizers $A^{(2)}, \ldots, A^{(n)}$ are well defined. $R$ is called $a G L_{q}(n)$ type R-matrix if two conditions

$$
A^{(n)}\left(\frac{q^{n}}{n_{q}} I-R_{n}\right) A^{(n)}=0
$$

and

$$
\operatorname{rk} A^{(n)}=1
$$

are fulfilled.

Remark 2.8. Assuming $(n+1)_{q} \neq 0$, the condition $(2.4 .1)$ is equivalent to $A^{(n+1)}=0$. For generic values of $q$, assuming validity of (2.4.1), the condition (2.4.2) is equivalent to demanding skew invertibility of $R$ (see [G], Props. 3.6 and 3.10).

Proposition 2.9 [G,Is.04]. Let $R$ be a skew invertible R-matrix of the type $G L_{q}(n)$. Then $C_{R}$ and $D_{R}$ are invertible and the following relations are fulfilled:

$$
\begin{aligned}
& D_{R} C_{R}=C_{R} D_{R}=q^{-2 n} I, \\
& \operatorname{Tr}_{R}(k) A^{(k)}=q^{-n} \frac{(n+1-k)_{q}}{k_{q}} A^{(k-1)} \forall k=1,2, \ldots, n, \\
& A^{(n)} \prod_{i=1}^{n}\left(D_{R}\right)_{i}=\prod_{i=1}^{n}\left(D_{R}\right)_{i} A^{(n)}=q^{-n^{2}} A^{(n)} .
\end{aligned}
$$

Example 2.10. Consider the case $\mathfrak{A}_{\mathcal{R}}$ is the quantized universal enveloping algebra $U_{q} \mathfrak{s l}(n)$. Let $V$ be a vector representation of $U_{q} \mathfrak{s l}(n), \operatorname{dim} V=n$. In this case formula (2.2.11) with the scaling factor chosen as $\eta=q^{1 / n}$ gives a standard Drinfeld-Jimbo's R-matrix $R^{\circ}$ of the $G L_{q}(n)$ type (see [KSch], Sect. 8.4.2):

$$
R^{\circ}=\sum_{i, j=1}^{n} q^{\delta_{i j}} E_{i j} \otimes E_{j i}+\left(q-q^{-1}\right) \sum_{i<j} E_{i i} \otimes E_{j j} .
$$

Here $\left(E_{i j}\right)_{k l}:=\delta_{i k} \delta_{j l}, i, j=1, \ldots, n$, is a standard basis of $n \times n$ matrix units. Via the so-called twist procedure (for details see [R.90]) $R^{\circ}$ gives rise to a multiparametric family of $G L_{q}(n)$ type R-matrices,

$$
\begin{aligned}
& R^{f}:=F R^{\circ} F^{-1}=\sum_{i, j=1}^{n} q^{\delta_{i j}} \frac{f_{i j}}{f_{j i}} E_{i j} \otimes E_{j i}+\left(q-q^{-1}\right) \sum_{i<j} E_{i i} \otimes E_{j j}, \\
& \forall f_{i j} \in\{\mathbb{C} \backslash 0\} .
\end{aligned}
$$

Here $F:=\sum_{i, j=1}^{n} f_{i j} E_{i i} \otimes E_{j j}$ is a twisting $R$-matrix. In what follows we use these particular R-matrices for illustration purposes. Their corresponding matrices $D_{R^{\circ}}$ and $D_{R^{f}}$ are

$$
D_{R^{\circ}}=D_{R^{f}}=\sum_{i=1}^{n} q^{2(i-n)-1} E_{i i}
$$


Remark 2.11. Generally speaking, a $G L_{q}(n)$ type R-matrix can be realized in a tensor square of space $V$ whose dimension is different from $n$. Examples of the R-matrices for any $\operatorname{dim} V \geq n$ are given in [G], in Sect. 4. In what follows we do not assume any relation between the parameter $n$ in Definition 2.7 and the dimension of the space $V$, unless it is stated explicitly.

\section{Quantized Functions on a Cotangent Bundle Over Matrix Group}

In this section we recall the definition of a quantum group cotangent bundle and develop in linear cases $-G L_{q}(n)$ and $S L_{q}(n)$ - basic techniques for its structure investigation.

\subsection{Quantized functions over matrix group (RTT algebra).}

Definition 3.1 [D.86,FRT]. Let $R$ be a skew invertible R-matrix. An associative unital algebra generated by a set of matrix components $\left\|T_{j}^{i}\right\|_{i, j=1}^{\operatorname{dim} V}$ satisfying relations

$$
R_{12} T_{1} T_{2}=T_{1} T_{2} R_{12}
$$

is denoted as $\mathfrak{F}[R]$ and called an RTT algebra. The RTT algebra is endowed in a standard way with the coproduct and the counit

$$
\Delta\left(T_{j}^{i}\right)=\sum_{k} T_{k}^{i} \otimes T_{j}^{k}, \quad \epsilon\left(T_{j}^{i}\right)=\delta_{j}^{i} .
$$

Let further extend the RTT algebra by a set of inverse matrix components $\left\|\left(T^{-1}\right)_{j}^{i}\right\|_{i, j=1}^{\operatorname{dim} V}$ :

$$
\sum_{k} T_{k}^{i}\left(T^{-1}\right)_{j}^{k}=\sum_{k}\left(T^{-1}\right)_{k}^{i} T_{j}^{k}=\delta_{j}^{i} 1 .
$$

The extended algebra can be endowed with the antipode mapping

$$
S\left(T_{j}^{i}\right)=\left(T^{-1}\right)_{j}^{i}, \quad \text { so that (see [R.89]): } \quad S^{2}(T) D_{R}=D_{R} T .
$$

The resulting Hopf algebra is further denoted as $\mathfrak{F} \mathfrak{G}[R]$.

Example 3.2. Consider the quasi-triangular Hopf algebra $\mathfrak{A}_{\mathcal{R}}$ together with its representation $\rho_{V}$ (see Example 2.5). For any $x \in \mathfrak{A}_{\mathcal{R}}$ denote $\left\|\rho_{V}(x)_{j}^{i}\right\|$ a matrix of the operator $\rho_{V}(x)$ in a certain basis in the space $V$.

Let $\mathfrak{A}_{\mathcal{R}}^{*}$ be the dual Hopf algebra and let $\langle\cdot, \cdot\rangle$ denote a non degenerate pairing between $\mathfrak{A}_{\mathcal{R}}$ and $\mathfrak{A}_{\mathcal{R}}^{*}$. Consider two matrices of linear functionals on $\mathfrak{A}_{\mathcal{R}}-T_{j}^{i}$ and $\left(T^{-1}\right)_{j}^{i}-$ such that

$$
\left\langle T_{j}^{i}, x\right\rangle=\rho_{V}(x)_{j}^{i}, \quad\left\langle\left(T^{-1}\right)_{j}^{i}, x\right\rangle=\rho_{V}(S(x))_{j}^{i} \quad \forall x \in \mathfrak{A}_{\mathcal{R}} .
$$

It is easy to see that these functionals satisfy conditions of Definition 3.1 (for details see, e.g., [B]), the numeric R-matrix $R$ in (3.1.1) in this case is given by (2.2.11), relation (3.1.4) for the square of antipode descends from (2.1.4). The functionals $T_{j}^{i}$ and $\left(T^{-1}\right)_{j}^{i}$ generate a Hopf subalgebra in $\mathfrak{A}_{\mathcal{R}}^{*}$.

In case $\mathfrak{A}_{\mathcal{R}}$ is a universal enveloping algebra $U \mathfrak{g}$ of some Lie algebra $\mathfrak{g}$, the dual Hopf algebra $(U \mathfrak{g})^{*}$ can be treated as $\operatorname{Fun}(\mathfrak{G}) \equiv \mathfrak{F} \mathfrak{G}$, where $\mathfrak{G}$ is a formal group corresponding to $\mathfrak{g}$. Therefore, heuristically we can treat the RTT algebras $\mathfrak{F} \mathfrak{G}[R]$ and $\mathfrak{F}[R]$ as algebras of quantized functions over the matrix group and matrix semigroup, respectively. Here the term matrix refers to a matrix form of the coproduct (3.1.2); the term quantized means that relations (3.1.1) in general define a noncommutative product. 
In the rest of the subsection we describe a construction of the inverse matrix $T^{-1}$ for the RTT algebra associated with the $G L_{q}(n)$ type R-matrix.

Consider an element

$$
\operatorname{det}_{R} T:=\operatorname{Tr}_{(1, \ldots, n)}\left(A^{(n)} T_{1} T_{2} \ldots T_{n}\right)
$$

By the definition of the coproduct (3.1.2) and due to the rank 1 condition (2.4.2) the element $\operatorname{det}_{R} T$ is group-like

$$
\Delta\left(\operatorname{det}_{R} T\right)=\operatorname{det}_{R} T \otimes \operatorname{det}_{R} T,
$$

and it satisfies relations

$$
A^{(n)} T_{1} T_{2} \ldots T_{n}=T_{1} T_{2} \ldots T_{n} A^{(n)}=A^{(n)} \operatorname{det}_{R} T
$$

Therefore, it is natural to call $\operatorname{det}_{R} T$ a determinant of the matrix $T$.

Proposition 3.3 [G]. Let $R$ be a skew invertible $G L_{q}(n)$ type R-matrix. The following relation is satisfied in the corresponding RTT algebra $\mathfrak{F}[R]$ :

$$
\left(\operatorname{det}_{R} T\right) T=\left(O_{R} T \sigma_{R}^{1}\right) \operatorname{det}_{R} T,
$$

where $O_{R}, O_{R}^{-1} \in \operatorname{Aut}(V)$ are mutually inverse matrices:

$$
\begin{aligned}
O_{R 1} & =n_{q} \operatorname{Tr}_{(2, \ldots, n+1)}\left(P_{1} P_{2} \ldots P_{n} A^{(n)}\right), \\
\left(O_{R}^{-1}\right)_{1} & =n_{q} \operatorname{Tr}_{(2, \ldots, n+1)}\left(A^{(n)} P_{n} \ldots P_{2} P_{1}\right),
\end{aligned}
$$

(recall that $P_{i}$ are permutation operators acting in components spaces $V_{i} \otimes V_{i+1}$ ).

Corollary 3.4. In the assumptions of Proposition 3.3 consider an extension of the RTT algebra $\mathfrak{F}[R]$ by an element $\left(\operatorname{det}_{R} T\right)^{-1}$ subject to relations

$$
\left(\operatorname{det}_{R} T\right)^{-1} T=\left(O_{R}^{-1} T O_{R}\right)\left(\operatorname{det}_{R} T\right)^{-1}, \quad \operatorname{det}_{R} T\left(\operatorname{det}_{R} T\right)^{-1}=\left(\operatorname{det}_{R} T\right)^{-1} \operatorname{det}_{R} T=1 .
$$

In the extended algebra the inverse matrix $T^{-1}$ satisfying relations (3.1.3) is given by formula

$$
\left(T^{-1}\right)_{1}=q^{n(n-1)} n_{q} \operatorname{Tr}_{R}(2, \ldots, n)\left(T_{2} \ldots T_{n} A^{(n)}\right)\left(\operatorname{det}_{R} T\right)^{-1}
$$

The resulting Hopf algebra is called a $G L_{q}(n)$ type RTT algebra and denoted as $\mathfrak{F} G L_{q}(n)[R]$.

Assume additionally that for the R-matrix $R$ the corresponding matrix $O_{R}$ (3.1.7) is scalar: $O_{R} \propto I$. In this case $R$ is called the R-matrix of $S L_{q}(n)$ type. In the corresponding RTT algebra $\mathfrak{F}_{G L_{q}(n)_{R}}$ the element $\operatorname{det}_{R} T$ is central. A quotient of this algebra by relation $\operatorname{det}_{R} T=1$ is called $S L_{q}(n)$ type RTT algebra and denoted as $\mathfrak{F} S L_{q}(n)[R]$. 
Remark 3.5. For a skew invertible $G L_{q}(n)$ type R-matrix $R$ consider a system of equations

$$
R_{12} N_{1} N_{2}=N_{1} N_{2} R_{12}, \quad N^{n} \propto O_{R} \quad \text { for some } N \in \operatorname{Aut}(V) .
$$

Note that a consistency condition for these equations $-R_{12} O_{R 1} O_{R 2}=O_{R 1} O_{R 2} R_{12}-$ is satisfied (see [OP.05]). By any solution $N$ of these equations one can construct the $S L_{q}(n)$ type R-matrix

$$
\widetilde{R}_{12}:=N_{1} R_{12} N_{1}^{-1}=N_{2}^{-1} R_{12} N_{2} .
$$

Example 3.6. For the R-matrices described in Example 2.10 one has

$$
O_{R^{\circ}}=-I, \quad O_{R^{f}}=-\sum_{i=1}^{n}\left(\prod_{j \neq i} f_{j i} / f_{i j}\right) E_{i i}
$$

So, $R^{\circ}$ is $S L_{q}(n)$ type, while $R^{f}$ is $S L_{q}(n)$ type only if $\forall i=1, \ldots, n: \prod_{j \neq i}\left(f_{j i} / f_{i j}\right)$ $={ }^{n} \sqrt{1}$. Taking a diagonal $n^{\text {th }}$ root $O_{R^{f}}^{1 / n}$ of the diagonal matrix $O_{R^{f}}$ one finds the $S L_{q}(n)$ type R-matrix associated with $R^{f}$ :

$$
\tilde{R}^{f}=R^{\tilde{f}}, \text { where } \tilde{f}_{i j}:=\prod_{k \neq i, j}\left(f_{i j} f_{j k} f_{k i}\right)^{1 / n}, \quad \text { so that } \quad O_{R \tilde{f}}=-I .
$$

\subsection{Quantized right invariant vector fields (reflection equation algebra).}

Definition 3.7 [KS]. Let $R$ be a skew invertible R-matrix. An associative unital algebra $\mathfrak{L} \mathfrak{G}[R]$ generated by a set of matrix components $\left\|L_{j}^{i}\right\|_{i, j=1}^{\operatorname{dim} V}$ satisfying relations

$$
L_{1} R_{12} L_{1} R_{12}=R_{12} L_{1} R_{12} L_{1}
$$

is called a reflection equation algebra or, shortly, $\mathbf{R E}$ algebra. The RE algebra $\mathfrak{L} \mathfrak{G}[R]$ is naturally endowed with a structure of left coadjoint $\mathfrak{F} \mathfrak{G}[R]$-comodule algebra

$$
\delta_{\ell}\left(L_{j}^{i}\right)=\sum_{k, m} T_{k}^{i}\left(T^{-1}\right)_{j}^{m} \otimes L_{m}^{k} .
$$

Example 3.8. [FRT]. In notations of Examples 2.5, 3.2 consider the following $\mathfrak{A}_{\mathcal{R}}$-valued matrices

$$
\begin{gathered}
L_{j}^{(+)}{ }_{j}=\left\langle i d \otimes T_{j}^{i}, \mathcal{R}\right\rangle, \quad L_{j}^{(-)}=\left\langle S\left(T_{j}^{i}\right) \otimes i d, \mathcal{R}\right\rangle=\left\langle T_{j}^{i} \otimes i d, \mathcal{R}^{-1}\right\rangle, \\
\left(\left(L^{(+)}\right)^{-1}\right)_{j}^{i}=\left\langle i d \otimes T_{j}^{i}, \mathcal{R}^{-1}\right\rangle, \quad\left(\left(L^{(-)}\right)^{-1}\right)_{j}^{i}=\left\langle T_{j}^{i} \otimes i d, \mathcal{R}\right\rangle .
\end{gathered}
$$

As a consequence of the Yang-Baxter equation (2.1.3) components of these matrices satisfy relations

$$
R_{12} L_{2}^{( \pm)} L_{1}^{( \pm)}=L_{2}^{( \pm)} L_{1}^{( \pm)} R_{12}, \quad R_{12} L_{2}^{(+)} L_{1}^{(-)}=L_{2}^{(-)} L_{1}^{(+)} R_{12}
$$

where $R$ is given by (2.2.11). By (2.1.2), the elements $\left(\left(L^{( \pm)}\right)^{ \pm 1}\right)_{j}^{i}$ generate a Hopf $\mathfrak{A}_{\mathcal{R}}$-subalgebra

$$
\Delta\left(L_{j}^{( \pm)}{ }_{j}^{i}\right)=\sum_{k} L_{k}^{( \pm)} \otimes L_{j}^{( \pm)}, \quad \epsilon\left(L_{j}^{( \pm)}{ }_{j}^{i}\right)=\delta_{j}^{i}, \quad S\left(L_{j}^{( \pm)}{ }_{j}^{i}\right)=\left(\left(L^{( \pm)}\right)^{-1}\right)_{j}^{i} .
$$


Consider a composite matrix $L$ with components

$$
L_{j}^{i}:=q^{\left(n-\frac{1}{n}\right)} \sum_{k}\left(\left(L^{(-)}\right)^{-1}\right)_{k}^{i} L_{j}^{(+)}{ }_{j}=q^{\left(n-\frac{1}{n}\right)}\left\langle i d \otimes T_{j}^{i}, \mathcal{R}_{21} \mathcal{R}_{12}\right\rangle,
$$

where our choice of a numeric factor $q^{n-\frac{1}{n}}$ is argued in Appendix A. By (3.2.4), components of $L$ satisfy reflection equation (3.2.1), where $R$ is given by (2.2.11). Note that an $\mathfrak{A}_{\mathcal{R}}$-subalgebra generated by the elements $L_{j}^{i}$ (3.2.5) does not carry a natural Hopf algebra structure. Instead, it obeys a coadjoint comodule algebra structure (3.2.2) with respect to the Hopf $\mathfrak{A}_{\mathcal{R}}^{*}$-subalgebra generated by the components of the matrices $T$ and $T^{-1}(3.1 .5)$.

Let us comment on a geometric interpretation of the RE algebra. In [FRT] the matrices $L^{( \pm)}$were used to develop an RTT type description for the quantized universal enveloping algebra $U_{q} \mathfrak{g}$. Consider the case $\mathfrak{g}=\mathfrak{s l}(n)$ and let $V$ be its vector representation. The corresponding $G L_{q}(n)$ type R-matrix $R$ is given in Example 2.10. Making a linear change of generators $L_{j}^{i} \rightarrow \ell_{j}^{i}$ :

$$
L_{j}^{i}=\delta_{j}^{i}+\left(q-q^{-1}\right) \ell_{j}^{i},
$$

and using the Hecke condition (2.3.6) the reflection equation (3.2.1), for $q^{2} \neq 1$, can be equivalently rewritten as

$$
\ell_{1} R_{12} \ell_{1} R_{12}-R_{12} \ell_{1} R_{12} \ell_{1}=R_{12} \ell_{1}-\ell_{1} R_{12} .
$$

In a "classical" limit $q \rightarrow 1$ the R-matrix (2.4.6) tends to the permutation and Eqs. (3.2.7) go into commutation relations for the basis of generators of the Lie algebra $\mathfrak{g l}(n)$,

$$
\left[\ell_{1}, \ell_{2}\right]=P_{12}\left(\ell_{1}-\ell_{2}\right) \text {. }
$$

Classically we can treat $\left\|\ell_{j}^{i}\right\|_{i, j=1}^{p}$ as a basis of right invariant vector fields on $G L(n)$. Transformation of these basic fields under the left transition by a group element $t \in G L(n)$ is given by formula (cf. with (3.2.2))

$$
\delta_{\ell}(t): \quad \ell_{j}^{i} \mapsto \sum_{k, m=1}^{n} t_{k}^{i} \ell_{m}^{k}\left(t^{-1}\right)_{j}^{m}, \quad \text { where } t_{j}^{i}:=\rho_{V}(t)_{j}^{i} .
$$

Extrapolating this interpretation to a "quantum" case $q \neq 1$ we call $\left\|L_{j}^{i}\right\|_{i, j=1}^{n}$ a basis of quantized right invariant vector fields over the matrix group.

It is technically convenient to introduce the notation

$$
\begin{aligned}
& L_{\overline{1}}:=L_{1}, \quad L_{\overline{k+1}}:=R_{k} L_{\bar{k}} R_{k}^{-1}, \\
& L_{\underline{1}}:=L_{1}, \quad L_{\underline{k+1}}:=R_{k}^{-1} L_{\underline{k}} R_{k} \quad \forall k \geq 1 .
\end{aligned}
$$

In terms of these R-copies $L_{\bar{k}}, L_{k}$ of the matrix $L$ the reflection equation (3.2.1) can be equivalently written in any of the following forms:

$$
R_{k} L_{\bar{k}} L_{\overline{k+1}}=L_{\bar{k}} L_{\overline{k+1}} R_{k}, \quad R_{k} L_{\underline{k+1}} L_{\underline{k}}=L_{\underline{k+1}} L_{\underline{k}} R_{k} \quad \forall k \geq 1 .
$$

Taking into account commutativity relations

$$
R_{i} L_{\bar{k}}=L_{\bar{k}} R_{i}, \quad R_{i} L_{\underline{k}}=L_{\underline{k}} R_{i} \quad \forall i, k: k \neq i, i+1,
$$


one sees that the R-copies $L_{\bar{k}}\left(L_{\underline{k}}\right)$ of the matrix $L$ in the RE algebra $\mathfrak{L} \mathfrak{G}[R]$ formally satisfy the same relations as the usual copies $T_{k}\left(T_{k}^{-1}\right)$ of the matrix $T\left(T^{-1}\right)$ in the RTT algebra $\mathfrak{F} \mathfrak{G}[R]$.

Matrix monomials in two different series of the R-copies satisfy relations

$$
L_{\overline{1}} L_{\overline{2}} \ldots L_{\bar{k}}=L_{\underline{k}} \ldots L_{\underline{2}} L_{1} \quad \forall k \geq 1 \text {. }
$$

For $k=2$ the equality (3.2.12) is identical to the reflection equation (3.2.1). For $k>2$ this equality follows by induction on $k$. Note that monomials (3.2.12) transform covariantly under the left coadjoint coaction (3.2.2),

$$
\delta_{\ell}\left(L_{\overline{1}} \ldots L_{\bar{k}}\right)=\left(T_{1} \ldots T_{k} \otimes 1\right)\left(1 \otimes L_{\overline{1}} \ldots L_{\bar{k}}\right)\left(S\left(T_{1} \ldots T_{k}\right) \otimes 1\right) .
$$

The following proposition goes back to Theorem 14 from [FRT] (see also [Is.04], Prop. 5).

Proposition 3.9. Let $R$ be a skew invertible R-matrix. For an element $x^{(k)} \in \mathbb{C}\left[\mathcal{B}_{k}\right]$ denote

$$
\operatorname{ch}\left(x^{(k)}\right):=\operatorname{Tr}_{R}(1 \ldots k)\left(X_{R}^{(k)} L_{\overline{1}} L_{\overline{2}} \ldots L_{\bar{k}}\right)
$$

where $X_{R}^{(k)}:=\rho_{R}\left(x^{(k)}\right) \in \operatorname{End}\left(V^{\otimes k}\right)$. Consider a linear subspace $\mathfrak{C h}[R] \subset \mathfrak{L} \mathfrak{G}[R]$ spanned by the unity and by elements $c h\left(x^{(k)}\right) \forall k \geq 1$ and $\forall x^{(k)} \in \mathbb{C}\left[\mathcal{B}_{k}\right]$. The space $\mathfrak{C h}[R]$ is a subalgebra of the center of the RE algebra $\mathfrak{L} \mathfrak{G}[R]$. It is called a characteristic subalgebra of the $R E$ algebra $\mathfrak{L} \mathfrak{G}[R]$. The characteristic subalgebra is invariant with respect to the left $\mathfrak{F} \mathfrak{G}[R]$ coadjoint coaction (3.2.2).

Proof. In a setting of the quasi-triangular Hopf algebras these statements were proved in [D.89, R.89] (see there Sect. 3 and Sect. 4, respectively). Below we prove the proposition in the RE algebra setting.

Consider an arbitrary element $\operatorname{ch}\left(x^{(k)}\right)$ of the characteristic subalgebra. We first prove the following version of the formula (3.2.14):

$$
\begin{aligned}
\operatorname{ch}\left(x^{(k)}\right) I_{1} & =\operatorname{Tr}_{R}(2, \ldots, k+1)\left(X_{R}^{(k) \uparrow 1} L_{\overline{2}} L_{\overline{3}} \ldots L_{\overline{k+1}}\right) \\
& =\operatorname{Tr}_{R}(2, \ldots, k+1)\left(X_{R}^{(k) \uparrow 1} L_{\underline{k+1}} \ldots L_{\underline{3}} L_{\underline{2}}\right) .
\end{aligned}
$$

Here the first equality results from a calculation

$$
\begin{aligned}
& \operatorname{Tr}_{R}(2, \ldots, k+1)\left(X_{R}^{(k) \uparrow 1} L_{\overline{2}} \ldots L_{\overline{k+1}}\right) \\
& \quad=\operatorname{Tr}_{R}(2, \ldots, k+1)\left(X_{R}^{(k) \uparrow 1} R_{1} \cdots R_{k} L_{\overline{1}} \ldots L_{\bar{k}} R_{k}^{-1} \cdots R_{1}^{-1}\right) \\
& \quad=\operatorname{Tr}_{R}(2, \ldots, k+1)\left(R_{1} \cdots R_{k}\left(X_{R}^{(k)} L_{\overline{1}} \ldots L_{\bar{k}}\right) R_{k}^{-1} \cdots R_{1}^{-1}\right) \\
& \quad=\ldots=\operatorname{Tr}_{R}(1, \ldots, k)\left(X_{R}^{(k)} L_{\overline{1}} L_{\overline{2}} \ldots L_{\bar{k}}\right),
\end{aligned}
$$

where in the last line we applied (2.2.10) $k$ times. To prove the second equality in (3.2.15) we first use the relation (3.2.12) and then perform similar transformations. 
With the use of (3.2.15) and (3.2.12) checking centrality of $c h\left(x^{(k)}\right)$ is straightforward:

$$
\begin{aligned}
L_{1} \operatorname{ch}\left(x^{(k)}\right) & =\operatorname{Tr}_{R}(2, \ldots, k+1)\left(X_{R}^{(k) \uparrow 1} L_{\overline{1}} L_{\overline{2}} L_{\overline{3}} \ldots L_{\overline{k+1}}\right) \\
& =\operatorname{Tr}_{R}(2, \ldots, k+1)\left(X_{R}^{(k) \uparrow 1} L_{\overline{k+1}} \ldots L_{\overline{2}} L_{\overline{1}}\right)=\operatorname{ch}\left(x^{(k)}\right) L_{1} .
\end{aligned}
$$

The invariance of $\operatorname{ch}\left(x^{(k)}\right)$ under the left $\mathfrak{F} \mathfrak{G}[R]$ coadjoint coaction follows immediately from (3.2.13) together with the relation (3.1.4) for the square of antipode.

Consider a series of elements of the RE algebra $\mathfrak{L} \mathfrak{G}[R]$,

$$
p_{i}:=\operatorname{Tr}_{R}\left(L^{i}\right), \quad i=1,2, \ldots .
$$

Further on they are called power sums. The following calculation

$$
L_{1} p_{i}=\operatorname{Tr}_{R}(2) L_{1} R_{12} L_{1}^{i} R_{12}^{-1}=\operatorname{Tr}_{R}(2) R_{12}^{-1} L_{1}^{i} R_{12} L_{1}=p_{i} L_{1},
$$

proves centrality of the power sums. Here in the first and the last equalities we use formula (2.2.10), and the second equality is a consequence of (3.2.1). Actually, the power sums belong to the characteristic subalgebra $\mathfrak{C h}[R]$ :

$$
p_{i}=\operatorname{ch}\left(\sigma_{i-1} \ldots \sigma_{2} \sigma_{1}\right),
$$

which is verified by a following transformation:

$$
\begin{aligned}
\operatorname{ch}\left(\sigma_{i-1} \ldots\right. & \left.\sigma_{2} \sigma_{1}\right)=\operatorname{Tr}_{R}(1, \ldots, i) \\
& =\operatorname{Tr}_{R}(1, \ldots, i) \\
& =\operatorname{Tr}_{R}\left(L_{\overline{1}} \ldots L_{\bar{i}} \ldots L_{\overline{i-1}}\left(R_{i-1} \ldots R_{i-1} \ldots R_{1}\right) L_{1}\left(R_{1}^{-1} \ldots R_{i-1}^{-1}\right)\left(R_{i-1} \ldots L_{\overline{i-1}}\left(\operatorname{Tr}_{R}(i) R_{i-1}\right)\left(R_{i-2} \ldots R_{1}\right) L_{1}\right)\right. \\
& =\operatorname{Tr}_{R}(1, \ldots, i-2) \\
& \left.=\ldots=L_{\overline{1}} \ldots L_{\overline{i-2}}\left(\operatorname{Tr}_{R}(i-1) R_{i-2}\right)\left(R_{i-3} \ldots R_{1}\right) L_{1}^{2}\right) \\
& \operatorname{Tr}_{R}\left(L^{i}\right)=p_{i} .
\end{aligned}
$$

Here we repeatedly expand the notation $L_{\bar{j}}=\left(R_{j-1} \ldots R_{1}\right) L_{1}\left(R_{1}^{-1} \ldots R_{j-1}^{-1}\right)$ for $j=i, \ldots, 2$, and use (2.2.8).

Let $R$ be a skew invertible R-matrix of the Hecke type. Assuming that conditions [k] (2.3.1) are fulfilled consider a series of elements $a_{i} \in \mathfrak{C h}[R], i=0,1, \ldots k$, in the corresponding Hecke type RE algebra $\mathfrak{L} \mathfrak{G}[R]$,

$$
a_{0}:=1, \quad a_{i}:=\operatorname{ch}\left(a^{(i)}\right)=\operatorname{Tr}_{R}(1, \ldots, i)\left(A^{(i)} L_{\overline{1}} \ldots L_{\bar{i}}\right) \quad \forall 1 \leq i \leq k,
$$

where notations $a^{(i)}, A^{(i)}$ were explained in (2.3.2), (2.3.8). The elements $a_{i}$ are called elementary symmetric functions.

Definition 3.10. Let $R$ be a skew invertible $G L_{q}(n)$ type R-matrix. A central extension of the corresponding RE algebra $\mathfrak{L} \mathfrak{G}[R]$ by an element $a_{n}^{-1}: a_{n} a_{n}^{-1}=1$ is called $G L_{q}(n)$ type $\mathbf{R E}$ algebra and denoted as $\mathfrak{L} G L_{q}(n)[R]$. A quotient of this algebra by a relation

$$
a_{n}=q^{-1} 1
$$

is called $S L_{q}(n)$ type $\mathbf{R E}$ algebra and denoted as $\mathfrak{L} S L_{q}(n)[R]$. 
Remark 3.11. An actual value of a numeric factor in the right-hand side of (3.2.18) is not relevant for the definition. Our choice allows avoiding numeric factors later in formula (4.1.1) (see the proof of Proposition 4.1).

Consider realization of the RE algebra $\mathfrak{L} S L_{q}(n)[R]$ as a subalgebra in the quasi-triangular Hopf algebra $\mathfrak{A}_{\mathcal{R}}$ (see Example 3.8). In this case the condition (3.2.18) is consistent with the pairing $\langle\cdot, \cdot\rangle$ of the dual Hopf algebras $\mathfrak{A}_{\mathcal{R}}$ and $\mathfrak{A}_{\mathcal{R}}^{*}$ only for the chosen normalizations (3.2.5) for $L$ and $\eta=q^{1 / n}$ for $R$ (2.2.11). This point is explained in Appendix A, see (A.3).

Remark 3.12. The $G L_{q}(n)$ type R-matrix $R$ and its $S L_{q}(n)$ partner R-matrix $\widetilde{R}(3.1 .8)$ define identical RE algebras.

In the theorem below we describe Cayley-Hamilton and Newton identities specific to the $G L_{q}(n)$ type and Hecke type RE algebras.

Theorem 3.13. Let $R$ be a skew invertible R-matrix of the Hecke type. Assume that the conditions [k] (2.3.1) are fulfilled. Then in the corresponding RE algebra $\mathfrak{L} \mathfrak{G}[R]$ the following Cayley-Hamilton-Newton identities [IOP.98,IOP.99]

$$
i_{q} \operatorname{Tr}_{R}(2, \ldots, i)\left(A^{(i)} L_{\overline{2}} L_{\overline{3}} \ldots L_{\bar{i}}\right)=(-1)^{i+1} \sum_{j=0}^{i-1}(-q)^{j} a_{j} L_{1}^{i-j-1} \quad \forall 2 \leq i \leq k
$$

take place. Multiplying by $L_{1}$ from the left and taking the $\mathrm{R}$-trace $\operatorname{Tr}_{R}(1)$ of these identities one obtains Newton relations for the sets of power sums $\left\{p_{i}\right\}_{i \geq 1}$ and the set of elementary symmetric functions $\left\{a_{i}\right\}_{i \geq 0}$ [GPS.97],

$$
i_{q} a_{i}+(-1)^{i} \sum_{j=0}^{i-1}(-q)^{j} a_{j} p_{i-j}=0 \quad \forall 1 \leq i \leq k .
$$

Both sets $\left\{1, p_{j}\right\}_{j \geq 1}$ and $\left\{a_{j}\right\}_{j \geq 0}$ in this case generate the characteristic subalgebra $\mathfrak{C h}[R]$.

Assume additionally that $R$ is an R-matrix of the $G L_{q}(n)$ type. Then the finite set $\left\{a_{i}\right\}_{i=0}^{n}$ generates the characteristic subalgebra of the RE algebra $\mathfrak{L}_{G L_{q}(n)}[R]$, and following Cayley-Hamilton identity is fulfilled [GPS.97]:

$$
\sum_{i=0}^{n}(-q)^{i} a_{i} L^{n-i}=0
$$

This identity leads, in particular, to an invertibility of the matrix L:

$$
L^{-1}=q^{-1} a_{n}^{-1} \sum_{i=0}^{n-1}(-q)^{-i} a_{n-i-1} L^{i} .
$$

Remark 3.14. One can introduce generating functions $a(x), p(x)$ for the elementary symmetric functions and for the power sums

$$
a(x):=\sum_{i \geq 0} a_{i} x^{i}, \quad p(x):=\sum_{i \geq 1} p_{i} x^{i} .
$$


The Newton relations (3.2.20) can be written as a finite difference equation for the generating functions

$$
a(q x) p(-x)=\frac{a\left(q^{-1} x\right)-a(q x)}{q-q^{-1}} .
$$

For the $G L_{q}(n)$ type RE algebra we now construct its central extension by roots of the characteristic polynomial (3.2.21).

Definition 3.15. Denote $\mathfrak{S}_{n} a \mathbb{C}$-algebra of polynomials in $n$ pairwise commuting invertible indeterminates $\mu_{\alpha}^{ \pm 1}$ and their differences $\left(\mu_{\alpha}-\mu_{\beta}\right)^{ \pm 1}, \alpha, \beta=1, \ldots, n, \alpha \neq \beta$.

Let $R$ be a skew invertible R-matrix of the $G L_{q}(n)$ type, $\mathfrak{L} G L_{q}(n)[R]$ be the corresponding $R E$ algebra, and $\mathfrak{C h}[R]$ be its characteristic subalgebra. Consider a monomorphism $\mathfrak{C h}[R] \hookrightarrow \mathfrak{S}_{n}$ defined on generators as 5

$$
a_{i} \mapsto e_{i}\left(\mu_{1}, \ldots, \mu_{n}\right):=\sum_{1 \leq j_{1}<\cdots<j_{i} \leq n} \mu_{j_{1}} \mu_{j_{2}} \ldots \mu_{j_{i}} \quad \forall i=0,1, \ldots, n,
$$

where $e_{i}$ are the elementary symmetric functions of their arguments. The map (3.2.22) defines naturally a structure of, say, left $\mathfrak{C h}[R]$-module on $\mathfrak{S}_{n}$. A central extension of the algebra $\mathfrak{L} G L_{q}(n)[R]$,

$$
\begin{aligned}
& \overline{\mathfrak{L}} G L_{q}(n)[R]:=\mathfrak{L} G L_{q}(n)[R] \underset{\mathfrak{C h}_{R}}{\bigotimes} \mathfrak{S}_{n}: \\
& a_{\alpha}=e_{\alpha}\left(\mu_{1}, \ldots, \mu_{n}\right), \quad L_{j}^{i} \mu_{\alpha}=\mu_{\alpha} L_{j}^{i} \quad \forall i, j=1, \ldots, \operatorname{dim} V, \quad \forall \alpha=1, \ldots, n,
\end{aligned}
$$

is called a (semisimple) spectral completion of $\mathfrak{L} G L_{q}(n)[R]$. A quotient of this algebra by relations

$$
a_{n}=\prod_{\alpha=1}^{n} \mu_{\alpha}=q^{-1}
$$

is called a (semisimple) spectral completion of $\mathfrak{L} S L_{q}(n)[R]$ and denoted as $\overline{\mathfrak{L}} S L_{q}(n)[R]$. Variables $\mu_{\alpha}$ are called spectral variables.

Remark 3.16. Assuming that the spectral variables $\mu_{\alpha}$ are invariants of the coadjoint coaction, the algebra $\overline{\mathfrak{L}} G L_{q}(n)[R]\left(\overline{\mathfrak{L}} S L_{q}(n)[R]\right)$ inherits the structure of left coadjoint $\mathfrak{F} G L_{q}(n)[R]-\left(\mathfrak{F} S L_{q}(n)[R]-\right)$ comodule algebra.

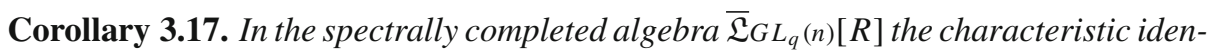
tity (3.2.21) assumes a factorized form

$$
\prod_{\alpha=1}^{n}\left(L-q \mu_{\alpha} I\right)=0 \text {. }
$$

\footnotetext{
5 When defining the map (3.2.22) we implicitly assume an algebraic independence of the elements $a_{i}$, $i=1, \ldots, n$. Otherwise, we should impose the same algebraic conditions on functions $e_{i}\left(\mu_{1}, \ldots, \mu_{n}\right)$.
} 
One can construct a resolution of the matrix unity

$$
P^{\alpha}:=\prod_{\substack{\beta=1 \\ \beta \neq \alpha}}^{n} \frac{\left(L-q \mu_{\beta} I\right)}{q\left(\mu_{\alpha}-\mu_{\beta}\right)}: P^{\alpha} P^{\beta}=\delta_{\alpha \beta} P^{\alpha}, \quad \sum_{\alpha=1}^{n} P^{\alpha}=I,
$$

so that

$$
L P^{\alpha}=P^{\alpha} L=q \mu_{\alpha} P^{\alpha} .
$$

Remark 3.18. In papers [GS.99,DM.01,DM.02,GS.04] the factorized form of the Cayley-Hamilton identity and the projectors $P^{\alpha}$ were used to construct explicitly quantized semisimple coadjoint orbits of $G L(n)$ and line bundles over them.

\subsection{Quantized differential operators over matrix group (Heisenberg double).}

Definition 3.19 [AF.91,S]. Let $R, T$ and $L$ be as described in Definitions 3.1 and 3.7. $A$ Heisenberg double (HD) algebra $\mathfrak{D G}[R, \gamma]$ of the two algebras $\mathfrak{F} \mathfrak{G}[R]$ and $\mathfrak{L} \mathfrak{G}[R]$ is an associative unital algebra generated by the components of the matrices $T$ and $L$ subject additionally to a permutation relation

$$
\gamma^{2} T_{1} L_{2}=R_{12} L_{1} R_{12} T_{1}, \text { where } \gamma \in\{\mathbb{C} \backslash 0\} .
$$

The HD algebra carries structures of left and right $\mathfrak{F} \mathfrak{G}[R]$-comodule algebra, respectively,

$$
\begin{aligned}
& \delta_{\ell}\left(T_{j}^{i}\right)=\sum_{k} T_{k}^{i} \otimes T_{j}^{k}, \quad \delta_{\ell}\left(L_{j}^{i}\right)=\sum_{k, m} T_{k}^{i}\left(T^{-1}\right)_{j}^{m} \otimes L_{m}^{k} ; \\
& \delta_{r}\left(T_{j}^{i}\right)=\sum_{k} T_{k}^{i} \otimes T_{j}^{k}, \quad \delta_{r}\left(L_{j}^{i}\right)=L_{j}^{i} \otimes 1 .
\end{aligned}
$$

Example 3.20. The Heisenberg double is closely related to a smash product of two mutually dual Hopf algebras (see, e.g., [M]). Namely, given a pair $\mathfrak{A}_{\mathcal{R}}$ and $\mathfrak{A}_{\mathcal{R}}^{*}$ their smash product algebra $\mathfrak{A}_{\mathcal{R}} \sharp \mathfrak{A}_{\mathcal{R}}^{*}$ is a linear space $\mathfrak{A}_{\mathcal{R}} \otimes \mathfrak{A}_{\mathcal{R}}^{*}$ supplied with a multiplication

$$
(x \sharp u)(y \sharp v):=\left\langle u_{(1)}, y_{(2)}\right\rangle\left(x y_{(1)} \sharp u_{(2)} v\right),
$$

where $x, y \in \mathfrak{A}_{\mathcal{R}}, u, v \in \mathfrak{A}_{\mathcal{R}}^{*}$, and symbols $(x \sharp u),(y \sharp v)$ denote elements of $\mathfrak{A}_{\mathcal{R}} \sharp \mathfrak{A}_{\mathcal{R}}^{*}$.

Let us calculate in the settings of Examples 3.2, 3.8 the smash product of the elements $\left(T_{j}^{i} \sharp 1\right)=T_{j}^{i}$ and $\left(1 \sharp L_{j}^{( \pm)}{ }_{j}\right)=L_{j}^{( \pm)}{ }_{j}$,

$$
\begin{aligned}
& T_{1} L_{2}^{(+)}=L_{2}^{(+)}\left\langle T_{1}, L_{2}^{(+)}\right\rangle T_{1}=\eta^{-1} L_{2}^{(+)} P_{12} R_{12} T_{1}, \\
& T_{1} L_{2}^{(-)}=L_{2}^{(-)}\left\langle T_{1}, L_{2}^{(-)}\right\rangle T_{1}=\eta L_{2}^{(-)} P_{12} R_{12}^{-1} T_{1},
\end{aligned}
$$

wherefrom it follows that the smash product of $T_{1}$ and $L_{2} \propto\left(L^{(-)}-1 L^{(+)}\right)_{2}$ is given by (3.3.1) with $\gamma=\eta$. However, we stress that in general one can keep $\gamma$ independent of the normalization $\eta$ of the R-matrix at the price of losing universality of formulas. Indeed, the multiplication in the smash product algebra is given by (3.3.4) universally for any pair of its elements, while the relation (3.3.1) in the HD algebra is written for the generators $L$ and $T$ only. 
Now we consider a geometric interpretation of the HD algebra. Applying the substitution $L_{j}^{i} \rightarrow \ell_{j}^{i}$ (3.2.6) and taking the "classical" limit $q \rightarrow 1$ in relation (3.3.1) we find

$$
\left[T_{1}, \ell_{2}\right]=\left(P_{12}-\gamma^{\prime} I_{12}\right) T_{1}
$$

where we used the Hecke condition (2.3.6) in a form $R^{2}=I+\left(q-q^{-1}\right) R$ and assumed additionally $R \stackrel{q \rightarrow 1}{\longrightarrow} P$ (which is true for the Drinfeld-Jimbo R-matrix (2.4.6)) and $\gamma \equiv \gamma(q)=1+\left(q-q^{-1}\right) \frac{\gamma^{\prime}}{2}+o\left(q-q^{-1}\right)$. The commutation relations (3.3.5), (3.2.8) are realized by operators

$$
\begin{gathered}
\ell_{j}^{i}=\sum_{k=1}^{n} g_{k}^{i} \frac{\partial}{\partial g_{k}^{j}}, \quad T_{j}^{i}=|g|^{-\gamma^{\prime}} g_{j}^{i}, \quad \text { where } g_{j}^{i}:=\rho_{V}(g)_{j}^{i}, g \in G L(n), \\
|g|:=\operatorname{det}\left\|g_{j}^{i}\right\| .
\end{gathered}
$$

These are, respectively, right invariant vector fields and properly normalized coordinate functions on $G L(n)$. Together they generate an algebra of differential operators over $G L(n)^{6}$.

Extrapolating the classical picture we can treat $\mathfrak{D} \mathfrak{G}[R, \gamma]$ as an algebra of quantized differential operators over the matrix group or, equivalently, as quantized functions over the cotangent bundle of a matrix group (see [AF.91,AF.92,SWZ.92,IP]). The form of the substitution (3.2.6) suggests that the quantized vector fields $L_{j}^{i}$ possess properties of finite difference operators rather than of the differential operators. In particular, they do not satisfy the classical Leibniz rule when acting on functions (see (3.3.1)).

The next proposition describes an action of the characteristic subalgebra on quantized functions in the Hecke case.

Proposition 3.21. Let $R$ be a skew invertible Hecke type R-matrix. Assume that the conditions [k] (2.3.1) are satisfied, so that the elementary symmetric functions $a_{i} \in \mathfrak{C h}[R] \subset \mathfrak{D G}[R, \gamma], 0 \leq i \leq k$, (3.2.17) are well defined. Then relations

$$
\gamma^{2 i} T a_{i}=a_{i} T-\left(q^{2}-1\right) \sum_{j=1}^{i}(-q)^{-j} a_{i-j}\left(L^{j} T\right) \quad \forall 0 \leq i \leq k
$$

are fulfilled for the Hecke type $\mathbf{H D}$ algebra $\mathfrak{D} \mathfrak{G}[R, \gamma]$.

Proof. For any operator $Y \in \operatorname{End}_{W}\left(V^{\otimes i}\right)$, where $W$ is an arbitrary $\mathbb{C}$-linear space, we denote

$$
Y^{\uparrow 1}:=\left(P_{1} P_{2} \ldots P_{i}\right) Y\left(P_{1} P_{2} \ldots P_{i}\right)^{-1}
$$

For any R-matrix $R$ we define series of operators $J_{i}, Z_{i}$,

$$
J_{1}:=I, \quad J_{i+1}:=R_{i} J_{i} R_{i} \quad \forall i \geq 1, \quad Z_{i}:=\prod_{j=1}^{i} J_{j} .
$$

6 Imposing conditions $\gamma^{\prime}=1 / n$, det $T=1, \operatorname{Tr} \ell=0$ one can make a reduction to a subalgebra of differential operators over $S L(n)$. 
Remark 3.22. Elements $J_{j}, 1 \leq j \leq i$, are R-matrix realizations of a remarkable set of Jucys-Murphy elements in the braid group $\mathcal{B}_{i}$ :

$$
j_{1}:=1, \quad j_{j+1}:=\sigma_{j} j_{j} \sigma_{j} \quad \forall j=1, \ldots, i-1 .
$$

These elements generate a commutative subgroup in $\mathcal{B}_{i}$ and their product $z_{i}:=\prod_{j=1}^{i} j_{j}$ is a central element in $\mathcal{B}_{i}$. For their applications and for historical references see, e.g., [OP.01].

With these notations permutation relations (3.2.1), (3.3.1) can be suitably written for arbitrary R-copies of the matrix $L$ :

$$
\begin{aligned}
\left(L_{\bar{i}} J_{i}\right)\left(L_{\bar{j}} J_{j}\right) & =\left(L_{\bar{j}} J_{j}\right)\left(L_{\bar{i}} J_{i}\right), \\
\gamma^{2} T_{1}\left(L_{\bar{i}} J_{i}\right)^{\uparrow 1} & =\left(L_{\bar{i}+1} J_{i+1}\right) T_{1}=R_{i}\left(L_{\bar{i}} J_{i}\right) R_{i} T_{1} \quad \forall i, j \geq 1 .
\end{aligned}
$$

Here the second equality follows from the recursive definitions of $L_{i+1}$ and $J_{i+1}$, while the first equality can be easily proved by induction on $i$.

Next, we prepare a suitable expression for $a_{i}$ (3.2.17):

$$
\begin{aligned}
a_{i} & =\operatorname{Tr}_{R}(1, \ldots, i)\left(L_{\overline{1}} \ldots L_{\bar{i}} A^{(i)}\right)=q^{i(i-1)} \operatorname{Tr}_{R}(1, \ldots, i)\left(L_{\overline{1}} \ldots L_{\bar{i}} Z_{i} A^{(i)}\right) \\
& =q^{i(i-1)} \operatorname{Tr}_{R}(1, \ldots, i)\left(\left(L_{\overline{1}} J_{1}\right) \ldots\left(L_{\bar{i}} J_{i}\right) A^{(i)}\right) .
\end{aligned}
$$

Here we substituted $Z_{i} A^{(i)}=q^{-i(i-1)} A^{(i)}$ in the first line and used a commutativity relation

$$
L_{\bar{i}} J_{j}=J_{j} L_{\bar{i}} \quad \forall i, j: i>j
$$

in the second line. By relabelling the subscript indices of the R-traces we then recast (3.3.11) in a following form ${ }^{7}$

$$
I_{1} a_{i}=q^{i(i-1)} \operatorname{Tr}_{R}(2, \ldots, i+1)\left(\left(L_{\overline{1}} J_{1}\right) \ldots\left(L_{\bar{i}} J_{i}\right) A^{(i)}\right)^{\uparrow 1} .
$$

Now we are ready to permute $T_{1}$ and $a_{i}$. Substituting expression (3.3.13) for $a_{i}$ and using relations (3.3.10) and (3.3.12) we calculate

$$
\begin{aligned}
\gamma^{2 i} T_{1} a_{i} & =\gamma^{2 i} T_{1}\left(I_{1} a_{i}\right)=q^{i(i-1)} \gamma^{2 i} \operatorname{Tr}_{R}(2, \ldots, i+1) T_{1}\left(\left(L_{\overline{1}} J_{1}\right) \ldots\left(L_{\bar{i}} J_{i}\right) A^{(i)}\right)^{\uparrow 1} \\
& =q^{i(i-1)} \operatorname{Tr}_{R}(2, \ldots, i+1)\left(\left(L_{\overline{2}} J_{2}\right) \ldots\left(L_{\overline{i+1}} J_{i+1}\right) A^{(i) \uparrow 1}\right) T_{1} \\
& =q^{i(i-1)} \operatorname{Tr}_{R}(2, \ldots, i+1) \\
& \left(\left(L_{\overline{2}} \ldots L_{\overline{i+1}}\right) Z_{i+1} A^{(i) \uparrow 1}\right) T_{1} .
\end{aligned}
$$

To continue the calculation we need the following formula:

$$
Z_{i+1} A^{(i) \uparrow 1}=A^{(i) \uparrow 1} Z_{i+1} A^{(i) \uparrow 1}=q^{-i(i-1)}\left(q^{2} A^{(i) \uparrow 1}-q^{-i}\left(q^{2}-1\right)(i+1)_{q} A^{(i+1)}\right),
$$

\footnotetext{
7 Notice a similarity of the formula (3.3.13) with the relation (2.2.10). The role of the R-matrices $R^{ \pm \varepsilon}$ is now played by the permutation matrix $P$ (see (3.3.7)).
} 
which follows by a combination of the definitions (2.3.3), (2.3.8), (3.3.8), and relations (2.3.4), (2.3.5), (2.3.6). So we finish the calculation

$$
\begin{aligned}
\gamma^{2 i} T_{1} a_{i} & =\operatorname{Tr}_{R}(2, \ldots, i+1)\left[\left(L_{\overline{2}} \ldots L_{i+1}\right)\left(q^{2} A^{(i) \uparrow 1}-q^{-i}\left(q^{2}-1\right)(i+1)_{q} A^{(i+1)}\right)\right] T_{1} \\
& =q^{2} a_{i} T_{1}+(-q)^{-i}\left(q^{2}-1\right) \sum_{j=0}^{i}(-q)^{-j} a_{j}\left(L^{i-j} T\right)_{1} \\
& =a_{i} T_{1}-\left(q^{2}-1\right) \sum_{j=1}^{i}(-q)^{-j} a_{i-j}\left(L^{j} T\right)_{1} .
\end{aligned}
$$

Here we calculate the first summand in the second line taking into account the equality

$$
\left(L_{\overline{2}} \ldots L_{\overline{i+1}}\right) A^{(i) \uparrow 1}=\left(R_{1} \ldots R_{i}\right)\left(L_{\overline{1}} \ldots L_{\bar{i}}\right) A^{(i)}\left(R_{1} \ldots R_{i}\right)^{-1}
$$

and using $i$ times formula (2.2.10). For calculation of the second summand we use the Cayley-Hamilton-Newton identity (3.2.19). Thus (3.3.6) is proved.

Remark 3.23. For the set of power sums (3.2.16) the permutation relations with $T_{j}^{i}$ in the Hecke case read

$$
\gamma^{2 i} T p_{i}=p_{i} T+\left(q-q^{-1}\right)^{2} \sum_{j=1}^{i-1} \frac{(2 j)_{q}}{2_{q}} p_{i-j}\left(L^{j} T\right)+\left(q-q^{-1}\right) \frac{(2 i)_{q}}{2_{q}}\left(L^{i} T\right) .
$$

One can derive this formula applying the R-trace $\operatorname{Tr}_{R}(2)$ to an equality $\gamma^{2 i} T_{1}\left(L_{2}\right)^{i}=$ $\left(R L_{1} R\right)^{i} T_{1}$ and taking into account relations

$$
\begin{aligned}
\left(R L_{1} R\right)^{i} & =R\left(L_{1}\right)^{i} R+\left(q-q^{-1}\right) \sum_{j=1}^{i-1} R^{2 j}\left(L_{1}\right)^{i-j} R\left(L_{1}\right)^{j}, \\
R^{2 j} & =2_{q}^{-1}\left(\left(q^{2 j-1}+q^{-2 j+1}\right) I+\left(q^{2 j}-q^{-2 j}\right) R\right) .
\end{aligned}
$$

These relations, in turn, follow inductively from the Hecke condition (2.3.6) and the reflection equation (3.2.1). Note that in this case there is no need to impose restrictions (2.3.1) on $q$.

Proposition 3.24. Let $R$ be a skew invertible $G L_{q}(n)$ type $\mathrm{R}$-matrix. An extension of the corresponding $H D$ algebra $\mathfrak{D G}[R, \gamma]$ by the elements $\left(\operatorname{det}_{R} T\right)^{-1}$ and $\left(a_{n}\right)^{-1}$, satisfying relations

$$
\begin{aligned}
\gamma^{2 n} L\left(\operatorname{det}_{R} T\right)^{-1} & =q^{2}\left(\operatorname{det}_{R} T\right)^{-1}\left(O_{R} L O_{R}^{-1}\right), \\
\gamma^{2 n}\left(a_{n}\right)^{-1} T & =q^{2} T\left(a_{n}\right)^{-1},
\end{aligned}
$$

in addition to those given in Definitions 3.4 and 3.10, is called $G L_{q}(n)$ type HD algebra and denoted as $\mathfrak{D} G L_{q}(n)[R, \gamma]$.

Let $R$ be a skew invertible $S L_{q}(n)$ type R-matrix. In the corresponding HD algebra $\mathfrak{D G}[R, \gamma]$ let us restrict the parameters by condition $\gamma^{n}=q$ and take a quotient by relations $\operatorname{det}_{R} T=1$ and $a_{n}=q^{-1} 1$. The quotient algebra is called $S L_{q}(n)$ type HD algebra and denoted as $\mathfrak{D} S L_{q}(n)[R]$. 
Remark 3.25. Notice consistency of the $S L_{q}(n)$ reduction condition $\gamma^{n}=q$ with the parameter restrictions $\eta=q^{1 / n}$ in Example 2.10 and $\gamma=\eta$ in Example 3.20.

Proof. Relations (3.3.15) and (3.3.16) should be consistent with permutation relations for $\operatorname{det}_{R} T$ and $a_{n}$ in the algebra $\mathfrak{D G}[R, \gamma]$. Permutation relations for $a_{n}$ with $T$ were in fact derived in the first line of the calculation (3.3.14) (put $i=n$ and take into account that $A^{(n+1)}=0$ in the $G L_{q}(n)$ case). The permutation relation for $\operatorname{det}_{R} T$ with $L$ can be derived by the same method as for $\operatorname{det}_{R} T$ with $T$ (see [G], Sect. 5, or [Is.04], calculation (3.5.39)). Given these results the consistency is obvious.

In the $S L_{q}(n)$ case $\left(O_{R} \propto I, \gamma^{n}=q\right)$ the elements $\operatorname{det}_{R} T$ and $a_{n}$ are central. Hence, $\mathfrak{D} S L_{q}(n)[R]$ is consistently defined.

Corollary 3.26. In the $G L_{q}(n)$ type HD algebra elements of the characteristic subalgebra satisfy the following commutation relations with $\operatorname{det}_{R} T$ :

$$
\gamma^{2 n k} \operatorname{det}_{R} T \operatorname{ch}\left(x^{(k)}\right)=q^{2 k} \operatorname{ch}\left(x^{(k)}\right) \operatorname{det}_{R} T \quad \forall x^{(k)} \in \mathcal{H}_{k}(q), k=1,2, \ldots
$$

Proof. A proof is a direct calculation of permutation of $c h\left(x^{(k)}\right)(3.2 .14)$ and $\operatorname{det}_{R} T$ exploiting relations (3.3.15) and properties of the matrix $O_{R}(3.1 .7)$,

$$
R_{12} O_{R 1} O_{R 2}=O_{R 1} O_{R 2} R_{12}, \quad O_{R} D_{R}=D_{R} O_{R} \text {. }
$$

The latter relations are proved in [OP.05], Sect. 5.3.

Theorem 3.27. Let $R$ be a skew invertible $G L_{q}(n)\left(S L_{q}(n)\right)$ type R-matrix. An extension of the corresponding $H D$ algebra $\mathfrak{D}_{G L_{q}(n)}[R, \gamma]\left(\mathfrak{D} S L_{q}(n)[R]\right)$ by the algebra $\mathfrak{S}_{n}$ of polynomials in mutually commuting indeterminates $\mu_{\alpha}^{ \pm 1},\left(\mu_{\alpha}-\mu_{\beta}\right)^{ \pm 1}$ satisfying relations (3.2.23) together with

$$
\gamma^{2}\left(P^{\beta} T\right) \mu_{\alpha}=q^{2 \delta_{\alpha \beta}} \mu_{\alpha}\left(P^{\beta} T\right) \quad \forall \alpha, \beta=1, \ldots, n,
$$

or, equivalently,

$$
\gamma^{2} T \mu_{\alpha}=\mu_{\alpha} T+\left(q^{2}-1\right) \mu_{\alpha}\left(P^{\alpha} T\right),
$$

is called a (semisimple) spectral completion of the $G L_{q}(n)\left(S L_{q}(n)\right)$ type HD algebra and denoted as $\overline{\mathfrak{D}} G L_{q}(n)[R, \gamma]\left(\overline{\mathfrak{D}} S L_{q}(n)[R]\right)$.

Remark 3.28. To avoid problems with permutations of $\left(\mu_{\alpha}-\mu_{\beta}\right)^{-1}$ with $P^{\sigma} T$ one could assume invertibility of all elements $\left(\mu_{\alpha}-q^{2 k} \mu_{\beta}\right) \forall \alpha \neq \beta, k \in \mathbb{Z}$. Further on we will not make such permutations and so we don't impose the corresponding restrictions.

Remark 3.29. Assuming that the spectral variables $\mu_{\alpha}$ are invariants of both left and right coactions, the algebra $\overline{\mathfrak{L}} G L_{q}(n)[R, \gamma]\left(\overline{\mathfrak{L}} S L_{q}(n)[R]\right)$ inherits the structures of left

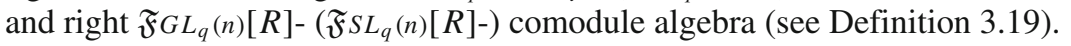

Remark 3.30. Note that relation (3.3.17) is typical for Weyl algebra generators. In fact there are many ways to combine from the elements $\left(P^{\beta} T\right)_{i j}$ a set of $n$ generators satisfying Weyl relations with the spectral variables $\mu_{\alpha}$. One such possibility is used later in Sect. 4.4. 
Proof. We have to check consistency of relations (3.3.17), (3.3.6) with the conditions $a_{i}=e_{i}\left(\mu_{1}, \ldots, \mu_{n}\right) \equiv e_{i}(\mu)$ for $1 \leq i \leq n$. Denote $e_{i}\left(\mu^{/ \alpha}\right):=\left.e_{i}(\mu)\right|_{\mu_{\alpha}=0}$. We have

$$
e_{i}(\mu)=e_{i}\left(\mu^{/ \alpha}\right)+\mu_{\alpha} e_{i-1}\left(\mu^{/ \alpha}\right) \Rightarrow e_{i}\left(\mu^{/ \alpha}\right)=\sum_{j=0}^{i}\left(-\mu_{\alpha}\right)^{j} e_{i-j}(\mu)
$$

Using relations (3.3.17), (3.3.18), (3.2.25) and (3.2.26) we calculate

$$
\begin{aligned}
\gamma^{2 i} T e_{i}(\mu) & =\gamma^{2 i} \sum_{\alpha=1}^{n}\left(P^{\alpha} T\right)\left(e_{i}\left(\mu^{/ \alpha}\right)+\mu_{\alpha} e_{i-1}\left(\mu^{/ \alpha}\right)\right) \\
& =\sum_{\alpha=1}^{n}\left(e_{i}\left(\mu^{/ \alpha}\right)+q^{2} \mu_{\alpha} e_{i-1}\left(\mu^{/ \alpha}\right)\right)\left(P^{\alpha} T\right) \\
& =\sum_{\alpha=1}^{n}\left(e_{i}(\mu)+\left(q^{2}-1\right) \mu_{\alpha} \sum_{j=0}^{i-1}\left(-\mu_{\alpha}\right)^{j} e_{i-j-1}(\mu)\right)\left(P^{\alpha} T\right) \\
& =\left(e_{i}(\mu)-\left(q^{2}-1\right) \sum_{j=1}^{i}(-L / q)^{j} e_{i-j}(\mu)\right) \sum_{\alpha=1}^{n}\left(P^{\alpha} T\right) \\
& =e_{i}(\mu) T-\left(q^{2}-1\right) \sum_{j=1}^{i}(-q)^{-j} e_{i-j}(\mu)\left(L^{j} T\right)
\end{aligned}
$$

which coincides with (3.3.6) under identification $e_{i}(\mu)=a_{i}$.

Corollary 3.31. In the completed $G L_{q}(n)$ type HD algebra $\overline{\mathfrak{D}} G L_{q}(n)[R, \gamma]$ the following permutation relations hold:

$$
\gamma^{2 n} \operatorname{det}_{R} T \mu_{\alpha}=q^{2} \mu_{\alpha} \operatorname{det}_{R} T \quad \forall \alpha=1,2, \ldots, n .
$$

Proof. Using formulas (3.1.6), (3.2.25), (3.3.17) we can permute $\operatorname{det}_{R} T$ and $\mu_{\alpha}$ :

$$
\begin{gathered}
\gamma^{2 n} \operatorname{det}_{R} T \mu_{\alpha}=\gamma^{2 n} \sum_{\beta_{1}, \ldots, \beta_{n}=1}^{n} \operatorname{Tr}_{(1, \ldots, n)}\left(A^{(n)}\left(P^{\beta_{1}} T\right)_{1} \ldots\left(P^{\beta_{n}} T\right)_{n}\right) \mu_{\alpha} \\
=\mu_{\alpha} \sum_{\beta_{1}, \ldots, \beta_{n}=1}^{n} q^{2 \sum_{j=1}^{n} \delta_{\alpha \beta_{j}}} \operatorname{Tr}_{(1, \ldots, n)}\left(A^{(n)}\left(P^{\beta_{1}} T\right)_{1} \ldots\left(P^{\beta_{n}} T\right)_{n}\right) .
\end{gathered}
$$

Assuming that

$\operatorname{Tr}_{(1, \ldots, n)}\left(A^{(n)}\left(P^{\beta_{1}} T\right)_{1} \ldots\left(P^{\beta_{n}} T\right)_{n}\right)=0, \quad$ if there exists a pair $i, j: \beta_{i}=\beta_{j}$,

we conclude that for any nonzero summand in (3.3.20) the coefficient $q^{2 \sum_{j=1}^{n} \delta_{\alpha \beta_{j}}}$ equals $q^{2}$, and therefore we can complete the calculation

$\gamma^{2 n} \operatorname{det}_{R} T \mu_{\alpha}=q^{2} \mu_{\alpha} \sum_{\beta_{1}, \ldots, \beta_{n}=1}^{n} \operatorname{Tr}_{(1, \ldots, n)}\left(A^{(n)}\left(P^{\beta_{1}} T\right)_{1} \ldots\left(P^{\beta_{n}} T\right)_{n}\right)=q^{2} \mu_{\alpha} \operatorname{det}_{R} T$. 
It remains to prove the assumption. First, we note that conditions on $\beta_{i}$ in (3.3.21) stand that there exists an integer $\sigma: 1 \leq \sigma \leq n$, and $\sigma \neq \beta_{i} \forall i$. Therefore, any projector $P^{\beta_{i}}$ in (3.3.21) contains the factor $\left(\bar{L}-\bar{q} \mu_{\sigma} I\right)$. Using relations (3.3.10), (3.3.17) we can move all such factors to the left side of the expression. Thus we obtain

$$
\text { left hand side of (3.3.21) } \propto \operatorname{Tr}_{(1, \ldots, n)}\left(A^{(n)}\left\{\prod_{j=1}^{n}\left(L_{j} J_{j}-q \mu_{\sigma} I\right)\right\} \ldots\right) \text {. }
$$

Next, we note that the expression in braces is a symmetric function in a commuting set of matrices $L_{\bar{j}} J_{j}$ (see (3.3.9)) which by relations

$$
\begin{aligned}
& R_{i}\left(L_{\bar{i}} J_{i}\right)\left(L_{\overline{i+1}} J_{i+1}\right)=\left(L_{\bar{i}} J_{i}\right)\left(L_{\overline{i+1}} J_{i+1}\right) R_{i}, \quad R_{i}\left(L_{\bar{i}} J_{i}+L_{\overline{i+1}} J_{i+1}\right) \\
& \quad=\left(L_{\bar{i}} J_{i}+L_{\overline{i+1}} J_{i+1}\right) R_{i},
\end{aligned}
$$

and by (3.2.11) together with the same formulas for $J_{k}$ commutes with $R_{i}, i=1, \ldots$, $n-1$, and so with $A^{(n)}$. Hence, using relations $A^{(n)}=\left(A^{(n)}\right)^{2}$ and $\operatorname{rk} A^{(n)}=1$ we can separate a left factor $\kappa:=\operatorname{Tr}_{(1, \ldots, n)}\left(A^{(n)} \prod_{j=1}^{n}\left(L_{\bar{j}} J_{j}-q \mu_{\sigma} I\right)\right)$ in (3.3.22). This factor we now calculate explicitly.

Taking into account relations $(2.4 .5),(3.3 .9),(3.3 .12)$ and $A^{(n)} J_{i}=q^{-2(i-1)} A^{(n)}$ we transform the expression for $\kappa$ :

$$
\kappa=q^{n} \operatorname{Tr}_{R}(1, \ldots, n)\left(A^{(n)} \prod_{j=1}^{n}\left(L_{\bar{j}}-q^{2 j-1} \mu_{\sigma} I\right)\right)
$$

Expanding this expression in powers of $L$ and noticing that (2.4.4) assumes $\operatorname{Tr}_{R}(k+1, \ldots, n) A^{(n)}=q^{n(k-n)} \frac{(k)_{q} !(n-k)_{q} !}{(n)_{q} !}:=q^{n(k-n)}\left(\begin{array}{l}n \\ k\end{array}\right)^{-1}$ we find that $k^{\text {th }}$ order monomials

$$
\operatorname{Tr}_{R}(1, \ldots, n)\left(A^{(n)} L_{\overline{i_{1}}} \ldots L_{\overline{i_{k}}}\right)=\operatorname{Tr}_{R}(1, \ldots, n)\left(A^{(n)} L_{\overline{1}} \ldots L_{\bar{k}}\right)=q^{n(k-n)}\left(\begin{array}{l}
n \\
k
\end{array}\right)_{q}^{-1} a_{k}
$$

are equal to each other for any choice of indices $1 \leq i_{1}<\ldots i_{k} \leq n$. Their corresponding coefficients in (3.3.23) sum up to

$$
\left(-q^{-1} \mu_{\sigma}\right)^{n-k} \sum_{1 \leq i_{1}<\ldots i_{n-k} \leq n} q^{2 \sum_{r=1}^{n-k} i_{r}}=q^{n(n-k)}\left(\begin{array}{l}
n \\
k
\end{array}\right)_{q}\left(-\mu_{\sigma}\right)^{n-k}
$$

and so we obtain

$$
\kappa=q^{n} \sum_{k=0}^{n} a_{k}\left(-\mu_{\sigma}\right)^{n-k}=q^{n} \prod_{\alpha=1}^{n}\left(\mu_{\alpha}-\mu_{\sigma}\right)=0
$$

where we took (3.2.23) into account. 
3.4. Quantized left invariant vector fields. In a classical differential geometry of the Lie groups one uses two global bases on tangent bundles - the bases of right and left invariant vector fields. In previous sections we discussed quantization of the right invariant vector fields only and defined the HD algebra $\mathfrak{D G}[R, \gamma]$ in their terms. To demonstrate a left-right symmetry of the whole construction we now describe the HD algebra using a set of left invariant generators. We also find explicit relations between the spectra of left and right invariant vector fields in both the $G L_{q}(n)$ and the $S L_{q}(n)$ cases.

In the assumptions of Definition 3.19 consider a matrix $M$ whose components belong to $\mathfrak{D} \mathfrak{G}[R, \gamma]$ :

$$
M_{j}^{i}:=\sum_{k, m}\left(T^{-1}\right)_{k}^{i} L_{m}^{k} T_{j}^{m}
$$

Taking into account transformation properties of the matrix elements $M_{j}^{i}$ with respect to the left and right $\mathfrak{F} \mathfrak{G}[R]$-coactions (3.3.2) and (3.3.3),

$$
\delta_{\ell}\left(M_{j}^{i}\right)=1 \otimes M_{j}^{i}, \quad \delta_{r}\left(M_{j}^{i}\right)=\sum_{k, m}\left(T^{-1}\right)_{k}^{i} T_{j}^{m} \otimes M_{m}^{k},
$$

we shall call them a basis of quantized left invariant vector fields over the matrix group.

One can give the presentation of the HD algebra $\mathfrak{D} \mathfrak{G}[R, \gamma]$ in terms of generators $T_{j}^{i}$ and $M_{j}^{i}$, and relations

$$
\begin{aligned}
\stackrel{*}{R_{12}} T_{2} T_{1} & =T_{2} T_{1} \stackrel{*}{R}_{12}, \\
\stackrel{*}{R} 12 M_{1} \stackrel{*}{R} 12 M_{1} & =M_{1} \stackrel{*}{R} M_{12} M_{1} \stackrel{*}{R} 12, \\
\gamma^{-2} M_{2} T_{1} & =T_{1} \stackrel{*}{R} M_{12} M_{1} \stackrel{*}{R}{ }_{12},
\end{aligned}
$$

where we denote

$$
\stackrel{*}{R} 12:=\left(P R^{-1} P\right)_{12}=\left(R_{21}\right)^{-1}
$$

Necessary technical data about $\stackrel{*}{R}$ are collected in Lemma B.1 in Appendix B.

By (3.4.3), the entries of matrix $M$ generate yet another RE subalgebra $\mathfrak{L} \mathfrak{G}[\stackrel{*}{R}]$ in the HD algebra $\mathfrak{D} \mathfrak{G}[R, \gamma]$. By (3.4.2), the subalgebra $\mathfrak{L} \mathfrak{G}[\stackrel{*}{R}]$ is a right coadjoint $\mathfrak{F} \mathfrak{G}[R]-$ comodule algebra. We also notice a nontrivial but quite expected property of the quantized left and right invariant vector fields - their mutual commutativity,

$$
M_{1} L_{2}=L_{2} M_{1} \text {. }
$$

In the rest of this section we investigate the characteristic subalgebra $\mathfrak{C h}[\stackrel{*}{R}] \subset$ $\mathfrak{L} \mathfrak{G}[\stackrel{*}{R}]$. In particular, we shall see that $\mathfrak{C h}[\stackrel{*}{R}]=\mathfrak{C} \mathfrak{h}[R]$ for the $\mathfrak{D} \mathfrak{G}[R, \gamma]$-subalgebras $\mathfrak{L} \mathfrak{G}[\stackrel{*}{R}]$ and $\mathfrak{L} \mathfrak{G}[R]$.

It is suitable to introduce $\stackrel{*}{R}$-copies of the matrix $M$ (cf. with (3.2.9))

$$
\left.M_{\frac{*}{1}}:=M_{1}, \quad M_{\frac{*}{k+1}}:=\stackrel{*}{R}_{k} M_{\frac{*}{k}} \stackrel{*}{R}\right)^{-1}
$$


and $\stackrel{*}{R}$-matrix realizations of the Jucys-Murphy elements (cf. with (3.3.8) and remark 3.22)

$$
\stackrel{*}{J}_{1}:=I, \quad \stackrel{*}{J}_{k+1}:=\stackrel{*}{R}_{k} \stackrel{*}{J}_{k} \stackrel{*}{R}_{k} \quad \forall k \geq 1 .
$$

In their terms the relations (3.4.3), (3.4.4) can be written as (cf. with (3.2.10), (3.3.10))

$$
\begin{aligned}
& \stackrel{*}{R} M_{\vec{*}} M_{\frac{*}{k+1}}=M_{\frac{*}{k}} M_{\frac{*}{k+1}} \stackrel{*}{R}, \\
& \gamma^{-2}\left(M_{\frac{*}{k}} \stackrel{*}{J}_{k}\right)^{\uparrow 1} T_{1}=T_{1}\left(M_{\frac{*}{k+1}} \stackrel{*}{J}_{k+1}\right) \text {. }
\end{aligned}
$$

We now assume that the R-matrix $\stackrel{*}{R}$ is skew invertible ${ }^{8}$ and introduce two generating sets in the characteristic subalgebra $\mathfrak{C h}[\stackrel{*}{R}] \subset \mathfrak{L} \mathfrak{G}[\stackrel{*}{R}]$ : the power sums $\stackrel{*}{p}_{k}$,

$$
\stackrel{*}{p}_{k}:=\operatorname{Tr}_{R}\left(M^{k}\right), \quad k=1,2, \ldots,
$$

and, assuming additionally that conditions [k] (2.3.1) are fulfilled, the elementary symmetric functions $\stackrel{*}{a}$,

$$
\stackrel{*}{a_{0}}:=1, \quad \stackrel{*}{a}{ }_{i}:=\operatorname{Tr}_{R}(1, \ldots, i)\left(\stackrel{*}{A}(i) M_{\frac{*}{1}} M_{\frac{*}{2}} \ldots M_{\frac{*}{i}}\right) \quad \forall 1 \leq i \leq k .
$$

Proposition 3.32. Let $R$ be a skew invertible Hecke type R-matrix and $D_{R}$ be invertible. Assume that conditions [k] (2.3.1) are satisfied. Then for two sets of elements in $\mathfrak{C h}[R] \subset \mathfrak{D} \mathfrak{G}[R, \gamma]-a_{i}(3.2 .17)$ and ${ }^{*}{ }_{i}(3.4 .9)$ - the following relations are satisfied

$$
\stackrel{*}{a}_{i}=\gamma^{2 i} a_{i} \quad \forall 0 \leq i \leq k .
$$

Proof. We transform the expression (3.4.9) for $\stackrel{*}{a}_{i}$ in the following way:

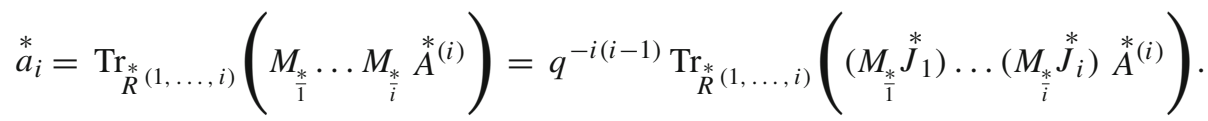

Here we used formulas

$$
\stackrel{*}{J_{k}} \stackrel{*}{A}^{(i)}=q^{2(k-1)} \stackrel{*}{A}^{(i)} \forall 1 \leq k \leq i, \quad \text { and } \quad M_{\frac{*}{\bar{i}}} \stackrel{*}{J}_{k}=\stackrel{*}{J}_{k} M_{\frac{*}{i}} \forall 1 \leq k<i,
$$

which, in turn, follow from (B.3), (3.4.6), (3.4.7).

Then we apply Lemma B.2 from Appendix B and use the relations (B.6) to move $\stackrel{*}{(i)}^{(i)}$ leftwards

$$
\begin{aligned}
\stackrel{*}{a}= & q^{-i(i-1)} \gamma^{i(i+1)} \operatorname{Tr}_{R}{ }_{R}(1, \ldots, i) \\
& \left.\times\left(T_{i} \ldots T_{1}\right)^{-1} \Upsilon_{P}^{(2 i)} \Upsilon_{P}^{(i)}\right) .
\end{aligned}
$$

Here matrices $\Upsilon_{*}^{(*)}$ are defined in (B.4).

\footnotetext{
8 This is indeed the case if $R$ is skew invertible and $D_{R}$ is invertible (see Lemma B.1 in Appendix B).
} 
Next, we permute $\stackrel{*}{A}^{(i) \uparrow i}$ with $\Upsilon_{*}^{(2 i)}$ and cancel terms $\Upsilon_{P}^{(i)} \Upsilon_{P}^{(2 i)}$ on the left and $\Upsilon_{P}^{(2 i)} \Upsilon_{P}^{(i)}$ on the right. The latter cancellation exchange the R-traces $\operatorname{Tr}_{R}$ and $\operatorname{Tr}_{R}$ :

$$
\begin{aligned}
\stackrel{*}{a}_{i}= & q^{-i(i-1)} \gamma^{i(i+1)} \operatorname{Tr}_{R}(1, \ldots, i) \operatorname{Tr}_{R}(i+1, \ldots, 2 i) \\
& \left((L T)_{i} \ldots(L T)_{1} \stackrel{*(i)}{\AA^{*}} \Upsilon_{R}^{(2 i)}\left(T_{i} \ldots T_{1}\right)^{-1}\right) .
\end{aligned}
$$

In the resulting expression all the R-traces $\operatorname{Tr}_{R}(i+1, \ldots, 2 i)$ can be evaluated with the help of Lemma B.3. So, we continue

$$
\begin{aligned}
\stackrel{*}{a_{i}} & =q^{i(i-1)} \gamma^{i(i+1)} \operatorname{Tr}_{R}(1, \ldots, i)\left((L T)_{i} \ldots(L T)_{1} \stackrel{*}{(i)}^{(i}\left(T_{i} \ldots T_{1}\right)^{-1}\right) \\
& =q^{i(i-1)} \gamma^{i(i+1)} \operatorname{Tr}_{R}(1, \ldots, i) \\
& \left.(L T)_{1} \ldots(L T)_{i}\left(T_{1} \ldots T_{i}\right)^{-1} A^{(i)}\right) .
\end{aligned}
$$

Here in the first line we used formula (B.14) and (3.4.10); in the second line we applied formula (B.2) and then, moved the two terms $\Upsilon_{P}^{(i)}$, respectively, to the left and to the right and cancelled them under the R-traces $\operatorname{Tr}_{R}(1, \ldots, i)$. Finally, using repeatedly permutation relations (3.3.10) and then formula (3.3.11) we complete the transformation

$$
\begin{aligned}
& \stackrel{*}{a_{i}}=q^{i(i-1)} \gamma^{i(i+1)} \operatorname{Tr}_{R}(1, \ldots, i) \\
& \quad\left((L T)_{1} \ldots(L T)_{i-1}\left(\left(L_{\overline{1}} J_{1}\right)^{\uparrow 1} T_{1}^{-1}\right)^{\uparrow(i-2)}\left(T_{1} \ldots T_{i-2}\right)^{-1} A^{(i)}\right) \\
& \quad \cdots=q^{i(i-1)} \gamma^{2 i} \operatorname{Tr}_{R}(1, \ldots, i)\left(\left(L_{\overline{1}} J_{1}\right) \ldots\left(L_{\bar{i}} J_{i}\right) A^{(i)}\right)=\gamma^{2 i} a_{i} .
\end{aligned}
$$

Remark 3.33. For the sets of power sums $p_{i}$ and $\stackrel{*}{p}_{i}$ one can prove following recurrent relations:

$$
\stackrel{*}{p_{i}}=\gamma^{2 i} p_{i}-\left(q-q^{-1}\right) \sum_{k=1}^{i-1} \gamma^{2 k} p_{k} \stackrel{*}{p}_{i-k}
$$

Corollary 3.34. Let $R$ be a skew invertible R-matrix of the $G L_{q}(n)$ type (in which case $D_{R}$ is invertible, see Proposition 2.9). Then for the matrix $M$ (3.4.1) generating the RE algebra $\mathfrak{L} G L_{q}(n)[\stackrel{*}{R}] \subset \mathfrak{D}_{G L_{q}(n)}[R, \gamma]$ the following Cayley-Hamilton identity is valid:

$$
\sum_{i=0}^{n}(-1 / q)^{i} \stackrel{*}{a}_{i} \quad M^{n-i}=\sum_{i=0}^{n}\left(-\gamma^{2} / q\right)^{i} a_{i} M^{n-i}=0
$$

In the spectrally completed algebra $\overline{\mathfrak{L}} G L_{q}(n)[\stackrel{*}{R}] \subset \overline{\mathfrak{D}} G L_{q}(n)[R, \gamma]$ this identity assumes a completely factorized form

$$
\prod_{\alpha=1}^{n}\left(M-\frac{\gamma^{2} \mu_{\alpha}}{q} I\right)=0 .
$$


With the factorized characteristic identity (3.4.11) one can construct yet another resolution of matrix unity (cf. with (3.2.25))

$$
S^{\alpha}:=\prod_{\substack{\beta=1 \\ \beta \neq \alpha}}^{n} \frac{\left(M-\gamma^{2} q^{-1} \mu_{\beta} I\right)}{\gamma^{2} q^{-1}\left(\mu_{\alpha}-\mu_{\beta}\right)}: S^{\alpha} S^{\beta}=\delta_{\alpha \beta} S^{\alpha}, \quad \sum_{\alpha=1}^{n} S^{\alpha}=I,
$$

so that

$$
M S^{\alpha}=S^{\alpha} M=\gamma^{2} q^{-1} \mu_{\alpha} S^{\alpha} .
$$

The relation between the two sets of projectors $P^{\alpha}$ and $S^{\alpha}$ is explained in the following proposition.

Proposition 3.35. In the spectrally completed algebra $\overline{\mathfrak{D}}_{G L_{q}(n)}[R, \gamma]\left(\overline{\mathfrak{D}}_{S L_{q}(n)}[R]\right)$ one has

$$
P^{\alpha} T S^{\beta}=\delta_{\alpha \beta} P^{\alpha} T \quad \text { or, equivalently, } \quad P^{\alpha} T=T S^{\alpha} .
$$

Proof. Taking into account relations $T M=L T$, (3.2.26) and (3.3.17) one finds

$$
P^{\alpha} T M=P^{\alpha} L T=q \mu_{\alpha}\left(P^{\alpha} T\right)=\left(P^{\alpha} T\right) \gamma^{2} q^{-1} \mu_{\alpha} .
$$

Hence, in view of (3.4.12),

$$
P^{\alpha} T S^{\beta}=P^{\alpha} T \prod_{\sigma \neq \beta} \frac{\gamma^{2} q^{-1}\left(\mu_{\alpha}-\mu_{\sigma}\right)}{\gamma^{2} q^{-1}\left(\mu_{\beta}-\mu_{\sigma}\right)} .
$$

In case $\alpha \neq \beta$ the factor with $\sigma=\alpha$ in the product vanishes. In case $\alpha=\beta$ (and so, $\sigma \neq \alpha)$ all the terms in the product are equal to 1 . So, the relation above reduces to the first equality in (3.4.13).

3.5. Derivation of dynamical R-matrix. In [AF.91] A.Alekseev and L.Faddeev used the dynamical R-matrix in their construction of the Heisenberg double algebra. Namely, they observed an appearance of the classical dynamical r-matrix in the Poisson relations for certain classical variables and then, by postulating a quantum counterpart of those relations, they derived defining formulas (as in Definition 3.19) for the algebra $\mathfrak{D} \mathfrak{G}[R, \gamma]$.

In this section we aim to explain an origin of the dynamical R-matrix in the context of the HD algebras. We show that the dynamical R-matrix $-R(\mu)_{\alpha \beta}$ - appears in the permutation relations for matrix components of the matrices

$$
W^{\alpha}:=P^{\alpha} T=T S^{\alpha},
$$

and the arguments of the dynamical R-matrix are just the spectral variables $\mu_{\alpha}$. In a sense, we solve an inverse problem to that considered in [AF.91].

Recall the definition of two projectors associated with the Hecke type R-matrix (see $(2.3 .6))$

$$
A^{(2)}=\frac{q I-R_{1}}{q+q^{-1}}, \quad S^{(2)}=\frac{q^{-1} I+R_{1}}{q+q^{-1}} .
$$

These projectors, called the antisymmetrizer and the symmetrizer, serve for suitable separation of the different eigenspaces of $R$. 
Theorem 3.36. In the completed HD algebra $\overline{\mathfrak{D}} G L_{q}(n)[R, \gamma]\left(\overline{\mathfrak{D}} S L_{q}(n)[R]\right)$ the matrices $W^{\alpha}$ (3.5.1) satisfy relations

$$
\begin{array}{ll}
S^{(2)}\left\{W_{1}^{\alpha} W_{2}^{\beta}+W_{1}^{\beta} W_{2}^{\alpha}\right\} A^{(2)}=A^{(2)}\left\{W_{1}^{\alpha} W_{2}^{\beta}+W_{1}^{\beta} W_{2}^{\alpha}\right\} S^{(2)}=0 & \forall \alpha, \beta, \\
S^{(2)}\left\{\left(\mu_{\beta}-q^{2} \mu_{\alpha}\right) W_{1}^{\alpha} W_{2}^{\beta}+\left(\mu_{\alpha}-q^{2} \mu_{\beta}\right) W_{1}^{\beta} W_{2}^{\alpha}\right\} S^{(2)}=0 & \forall \alpha \neq \beta, \\
A^{(2)}\left\{\left(\mu_{\alpha}-q^{2} \mu_{\beta}\right) W_{1}^{\alpha} W_{2}^{\beta}+\left(\mu_{\beta}-q^{2} \mu_{\alpha}\right) W_{1}^{\beta} W_{2}^{\alpha}\right. & \\
\left.-\left(q^{4}-1\right) \mu_{\alpha} \varphi_{\alpha \beta} W_{1}^{\alpha} W_{2}^{\alpha}-\left(q^{4}-1\right) \mu_{\beta} \varphi_{\beta \alpha} W_{1}^{\beta} W_{2}^{\beta}\right\} A^{(2)}=0 \quad \forall \alpha \neq \beta,
\end{array}
$$

where $\varphi_{\alpha \beta}:=\prod_{\sigma \neq \alpha, \beta} \frac{\mu_{\sigma}-q^{2} \mu_{\alpha}}{\mu_{\sigma}-\mu_{\beta}}$. Relations (3.5.3)-(3.5.4) and (3.3.17) (together with the appropriate conditions on the spectral variables $\left.\mu_{\alpha}\right)$ define the algebra

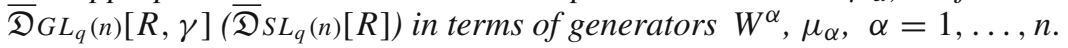

Proof. Consider the product $W_{1}^{\alpha} W_{2}^{\beta}$, where $\alpha \neq \beta$. With the help of (3.3.17) and (3.3.10) we can reorder terms of the product in a following way:

$$
\begin{aligned}
W_{1}^{\alpha} W_{2}^{\beta} & =\frac{\left(L_{1}-q \mu_{\beta}\right)\left(L_{\overline{2}} J_{2}-q^{3} \mu_{\alpha}\right)}{q^{2}\left(\mu_{\alpha}-\mu_{\beta}\right)\left(\mu_{\beta}-q^{2} \mu_{\alpha}\right)} W_{12}^{\alpha \beta}, \\
W_{12}^{\alpha \beta} & :=\prod_{\sigma \neq \alpha, \beta} \frac{\left(L_{1}-q \mu_{\sigma}\right)\left(L_{\overline{2}} J_{2}-q \mu_{\sigma}\right)}{q^{2}\left(\mu_{\alpha}-\mu_{\sigma}\right)\left(\mu_{\beta}-\mu_{\sigma}\right)} T_{1} T_{2} .
\end{aligned}
$$

Here factor $W_{12}^{\alpha \beta}$ commutes with the R-matrix $R_{12}$, which follows by the same arguments as in the proof of Corollary 3.31, see below (3.3.22). We now extract symmetric and antisymmetric parts of the product using projectors (3.5.2),

$$
\begin{aligned}
& S^{(2)} W_{1}^{\alpha} W_{2}^{\beta} \\
& =S^{(2)} \frac{L_{1} L_{\overline{2}} J_{2}+q^{4} \mu_{\alpha} \mu_{\beta} I-\frac{q^{2}\left(\mu_{\beta}+\mu_{\alpha}\right)}{q+q^{-1}}\left(L_{1}+L_{\overline{2}} J_{2}\right)+\frac{\mu_{\beta}-q^{2} \mu_{\alpha}}{q+q^{-1}}\left(L_{1}-L_{\overline{2}}\right)}{q^{2}\left(\mu_{\alpha}-\mu_{\beta}\right)\left(\mu_{\beta}-q^{2} \mu_{\alpha}\right)} W_{12}^{\alpha \beta},
\end{aligned}
$$

$$
\begin{aligned}
& A^{(2)} W_{1}^{\alpha} W_{2}^{\beta} \\
& =A^{(2)} \frac{L_{1} L_{\overline{2}} J_{2}+q^{4} \mu_{\alpha} \mu_{\beta} I-\frac{\mu_{\beta}+q^{4} \mu_{\alpha}}{q+q^{-1}}\left(L_{1}+L_{\overline{2}} J_{2}\right)+\frac{q^{2}\left(\mu_{\beta}-q^{2} \mu_{\alpha}\right)}{q+q^{-1}}\left(L_{1}-L_{\overline{2}}\right)}{q^{2}\left(\mu_{\alpha}-\mu_{\beta}\right)\left(\mu_{\beta}-q^{2} \mu_{\alpha}\right)} W_{12}^{\alpha \beta} .
\end{aligned}
$$

Here we separated linear in $L$ terms with the opposite symmetry properties

$$
R_{1}\left(L_{1}+L_{\overline{2}} J_{2}\right)=\left(L_{1}+L_{\overline{2}} J_{2}\right) R_{1}, \quad R_{1}\left(L_{1}-L_{\overline{2}}\right)=-\left(L_{1}-L_{\overline{2}}\right) R_{1}^{-1},
$$

which was done by the use of relation

$$
\begin{aligned}
q^{3} \mu_{\alpha} L_{1}+q \mu_{\beta} L_{\overline{2}} J_{2}= & \frac{q\left(I+R_{1}^{-2}\right)}{\left(q+q^{-1}\right)^{2}}\left\{\left(\mu_{\beta} R_{1}^{2}+q^{2} \mu_{\alpha} I\right)\left(L_{1}+L_{\overline{2}} J_{2}\right)\right. \\
& \left.+\left(q^{2} \mu_{\alpha}-\mu_{\beta}\right)\left(L_{1}-L_{\overline{2}}\right)\right\} .
\end{aligned}
$$


The symmetry properties (3.5.8) imply, in particular, that the only term contributing to expressions $A^{(2)} W_{1}^{\alpha} W_{2}^{\beta} S^{(2)}$ and $S^{(2)} W_{1}^{\alpha} W_{2}^{\beta} A^{(2)}$ is the one proportional to $\left(L_{1}-\right.$ $\left.L_{\overline{2}}\right)$, while the terms $\left(L_{1} L_{\overline{2}} J_{2}\right), I$ and $\left(L_{1}+L_{\overline{2}} J_{2}\right)$ contribute to $S^{(2)} W_{1}^{\alpha} W_{2}^{\beta} S^{(2)}$ and $A^{(2)} W_{1}^{\alpha} W_{2}^{\beta} A^{(2)}$.

It is now straightforward to check that formulas (3.5.3) and (3.5.4) follow from relations (3.5.6), (3.5.7). To check formula (3.5.5) one needs also similar expression for the product $W_{1}^{\alpha} W_{2}^{\alpha}$ :

$$
W_{1}^{\alpha} W_{2}^{\alpha}=\frac{L_{1} L_{\overline{2}} J_{2}+q^{2} \mu_{\beta}^{2} I-q \mu_{\beta}\left(L_{1}+L_{\overline{2}} J_{2}\right)}{q^{2} \varphi_{\alpha \beta}\left(\mu_{\alpha}-\mu_{\beta}\right)\left(q^{2} \mu_{\alpha}-\mu_{\beta}\right)} W_{12}^{\alpha \beta},
$$

and an analogous formula for $W_{1}^{\beta} W_{2}^{\beta}$. Here the factor $\varphi_{\alpha \beta}$ was defined in the proposition.

It remains to check that defining relations for the algebras $\overline{\mathfrak{D}}_{G L_{q}(n)}[R, \gamma]$ and $\overline{\mathfrak{D}} S L_{q}(n)[R]$ can be derived from (3.5.3)-(3.5.5) and (3.3.17). It is convenient to check relations for the matrices $T$ and $L T$ :

$$
\begin{aligned}
& R_{1} T_{1} T_{2}=T_{1} T_{2} R_{1}, \quad R_{1}(L T)_{1}(L T)_{2}=(L T)_{1}(L T)_{2} R_{1}, \\
& \quad \gamma^{2} T_{1}(L T)_{2}=R_{1}(L T)_{1} T_{2} R_{1} .
\end{aligned}
$$

For $G L_{q}(n)$ and $S L_{q}(n)$ types HD algebras, where $T$ is invertible, these formulas imply (3.2.1) and (3.3.1). Substituting expressions

$$
T=\sum_{\alpha=1}^{n} W^{\alpha}, \quad L T=q \sum_{\alpha=1}^{n} \mu_{\alpha} W^{\alpha},
$$

one can easily prove that the first two relations (3.5.9) follow from (3.5.3) and (3.3.17). Checking the last formula in (3.5.9) is also straightforward, although more lengthy. To this end one has to use the whole set of relations for $W$ 's and to take into account the identity $\sum_{\alpha \neq \beta} \varphi_{\alpha \beta}=1$.

Corollary 3.37. Relations (3.5.3)-(3.5.5) can be equivalently written as

$$
\begin{aligned}
& S^{(2)}\left[W_{1}^{\alpha} W_{2}^{\beta} R_{1}-\sum_{\alpha^{\prime}, \beta^{\prime}=1}^{n} R^{S}(q ; \mu)_{\alpha^{\prime} \beta^{\prime}}^{\alpha \beta} W_{1}^{\alpha^{\prime}} W_{2}^{\beta^{\prime}}\right]=0, \\
& A^{(2)}\left[W_{1}^{\alpha} W_{2}^{\beta} R_{1}-\sum_{\alpha^{\prime}, \beta^{\prime}=1}^{n} R^{A}(q ; \mu)_{\alpha^{\prime} \beta^{\prime}}^{\alpha \beta} W_{1}^{\alpha^{\prime}} W_{2}^{\beta^{\prime}}\right]=0,
\end{aligned}
$$

where $n^{2} \times n^{2}$ matrix $R^{S}(q ; \mu)$ has the following nonzero components:

$$
R_{\alpha \alpha}^{S_{\alpha \alpha}^{\alpha}}=q, \quad R_{\alpha \beta}^{S_{\beta}^{\alpha \beta}}=-\frac{\left(q-q^{-1}\right) \mu_{\beta}}{\mu_{\alpha}-\mu_{\beta}}, \quad R_{\beta \alpha}^{S_{\beta \beta}^{\alpha \beta}}=\frac{q^{-1} \mu_{\alpha}-q \mu_{\beta}}{\mu_{\alpha}-\mu_{\beta}} \forall \alpha \neq \beta,
$$

and $n^{2} \times n^{2}$ matrix $R^{A}(q ; \mu)$ has nonzero components at the same places as $R^{S}$ with values $R^{A}(q ; \mu)=R^{S}\left(-q^{-1} ; \mu\right)$, and the additional nonzero components

$$
R_{\beta \alpha}^{A \alpha}=-R_{\alpha \beta}^{A_{\alpha \alpha}^{\alpha \alpha}}=\frac{\left(q^{4}-1\right) \mu_{\alpha} \varphi_{\alpha \beta}}{q\left(\mu_{\alpha}-\mu_{\beta}\right)} \quad \forall \alpha \neq \beta .
$$


Both matrices $R^{S / A}(q ; \mu) \equiv R^{S / A}(\mu)$ satisfy the dynamical Yang-Baxter equation:

$$
R(\mu)^{12} R\left(\nabla^{1}(\mu)\right)^{23} R(\mu)^{12}=R\left(\nabla^{1}(\mu)\right)^{23} R(\mu)^{12} R\left(\nabla^{1}(\mu)\right)^{23} .
$$

Here superscript labels denote endomorphism spaces for the spectral indices, e.g., $R(\mu)_{\beta_{1} \beta_{2}}^{\alpha_{1} \alpha_{2}} \equiv R(\mu)^{12}$, and $\nabla^{1}$ is a diagonal finite shift operator,

$$
\nabla^{1}=\operatorname{diag}\left\{\nabla^{\alpha}\right\}_{\alpha=1}^{n}: \quad \nabla^{\alpha}\left(\mu_{\beta}\right):=q^{2 \delta_{\alpha \beta}} \gamma^{-2} \mu_{\beta} .
$$

Proof. Apply the symmetrizer $S^{(2)}$ and the antisymmetrizer $A^{(2)}$ from the right to both sides of the equalities $(3.5 .10),(3.5 .11)$. The resulting projections are easy to compare with (3.5.3)-(3.5.5).

To prove the dynamical Yang-Baxter equation for the matrices $R^{A}(q ; \mu)$ and $R^{S}(q ; \mu)$ we consider, respectively, the following cubic terms:

$$
A^{(3)} W_{1}^{\alpha} W_{2}^{\beta} W_{3}^{\sigma} \quad \text { and } \quad S^{(3)} W_{1}^{\alpha} W_{2}^{\beta} W_{3}^{\sigma} .
$$

Here the 3-antisymmetrizer $A^{(3)}=\rho_{R}\left(a^{(3)}\right)$ is the R-matrix realization of the idempotent $a^{(3)}$ (see (2.3.2), (2.3.3)), and the 3-symmetrizer $S^{(3)}$ is a similar projector which differs from $A^{(3)}$ by substitution $q \leftrightarrow-q^{-1}$ in the formulas (2.3.2), (2.3.3). Now applying two equal operators $R_{1} R_{2} R_{1}$ and $R_{2} R_{1} R_{2}$ from the right side to these terms and using relations (3.5.10), (3.5.11) and (3.3.17) one eventually proves (3.5.12) for $R^{A / S}(q ; \mu)$.

Remark 3.38. The dynamical R-matrix $R^{S}(q ; \mu)$ was constructed in [F.90,AF.91,Is.95]. A review on the dynamical Yang-Baxter equation and the dynamical R-matrices is given in [ES]. It is surprising that in our approach the dynamical R-matrices $R^{A / S}(q ; \mu)$, being the solutions of the nonlinear finite difference equation (3.5.12), are calculated by solving a system of (at most three) linear equations.

In concluding of the section we comment how relations (3.5.10) can be reduced to dynamical quadratic relations considered in [F.90,AF.91]. Recall that a (Hecke type) quantum plane $\mathcal{V}[R]$ is an algebra generated by components of vector $\left\{x_{i}\right\}_{i=1}^{\operatorname{dim} V}$ subject to relations

$$
x_{\langle 1|} x_{\langle 2|} A^{(2)}=0 \quad \Leftrightarrow \quad x_{\langle 1|} x_{\langle 2|} S^{(2)}=x_{\langle 1|} x_{\langle 2|} .
$$

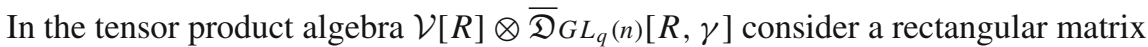

$$
\Lambda_{i}^{\alpha}:=\sum_{j=1}^{\operatorname{dim} V} x_{j} \otimes W_{j i}^{\alpha}, \quad \alpha=1, \ldots n, \quad i=1, \ldots, \operatorname{dim} V .
$$

As a consequence of (3.5.10), (3.5.14) the matrix components of $\Lambda$ fulfill relations

$$
\Lambda_{\langle 1|}^{|1\rangle} \Lambda_{\langle 2|}^{|2\rangle} R_{12}=R^{S}(q ; \mu)^{12} \Lambda_{\langle 1|}^{|1\rangle} \Lambda_{\langle 2|}^{|2\rangle} .
$$

Assume additionally that $i) \operatorname{dim} V=n$, and ii) the quantum plane admits a one dimensional representation $\chi: \mathcal{V}[R] \rightarrow \mathbb{C}$ (note that both these conditions are satisfied for the R-matrices from Example 2.10).

It is the square matrix $\chi(\Lambda) \in \overline{\mathfrak{D}} G L_{q}(n)[R, \gamma]$ whose dynamical quadratic relations (3.5.15) were introduced in [F.90,AF.91] and also investigated in [HIOPT, FHIOPT]. 


\section{Discrete Time Evolution on Quantum Group Cotangent Bundle}

4.1. Automorphisms of the Heisenberg double algebra. In this section we investigate a sequence of automorphisms on the HD algebra $\mathfrak{D} \mathfrak{G}[R, \gamma]$. These automorphisms were introduced by A. Alekseev and L. Faddeev [AF.91, AF.92], who interpreted them as a discrete time evolution of a $q$-deformed quantum isotropic Euler top. The automorphisms $\theta^{k}: \mathfrak{D} \mathfrak{G}[R, \gamma] \rightarrow \mathfrak{D} \mathfrak{G}[R, \gamma]$ are given on generators

$$
\begin{aligned}
& \{T, L\} \stackrel{\theta^{k}}{\longrightarrow}\{T(k), L(k)\}, \quad \forall k=0,1,2, \ldots, \\
& T(0):=T, \quad T(k+1):=L T(k)=L^{k+1} T, \quad L(k):=L .
\end{aligned}
$$

It is easy to see (cf. (3.5.9)) that the map $\theta$ agrees with the defining relations (3.1.1), (3.2.1), (3.3.1) of the algebra $\mathfrak{D G}[R, \gamma]$. Less obvious is its consistency with the $S L_{q}(n)$ type reduction conditions.

Proposition 4.1. The map $\theta$ (4.1.1) defines an automorphism of the algebra $\mathfrak{D} S L_{q}(n)[R]$.

Proof. It is necessary to check that $\operatorname{det}_{R}(L T)=1$ in the $S L_{q}(n)$ case. To this end, we use formula

$$
(L T)_{1}(L T)_{2} \ldots(L T)_{k}=\gamma^{-k(k-1)} Z_{k}\left(L_{\overline{1}} L_{\overline{2}} \ldots L_{\bar{k}}\right)\left(T_{1} T_{2} \ldots T_{k}\right)
$$

to separate matrices $L$ and $T$ in the expression for $\operatorname{det}_{R}(L T)$. This formula follows from (3.3.1), (3.2.10), (3.2.11) and (3.3.8) by induction on $k$.

The calculation of $\operatorname{det}_{R}(L T)$ proceeds as follows:

$$
\begin{aligned}
\operatorname{det}_{R} L T & :=\operatorname{Tr}_{(1, \ldots, n)}\left(A^{(n)}(L T)_{1} \ldots(L T)_{n}\right) \\
& =\gamma^{n(n-1)} \operatorname{Tr}_{(1, \ldots, n)}\left(A^{(n)} Z^{(n)} L_{\overline{1}} \ldots L_{\bar{n}} T_{1} \ldots T_{n}\right) \\
& =(\gamma q)^{-n(n-1)} \operatorname{Tr}_{(1, \ldots, n)}\left(A^{(n)} L_{\overline{1}} \ldots L_{\bar{n}}\right) \operatorname{Tr}_{(1, \ldots, n)}\left(A^{(n)} T_{1} \ldots T_{n}\right) \\
& =q^{n} \gamma^{-n(n-1)} \operatorname{Tr}_{R}(1, \ldots, n)\left(A^{(n)} L_{\overline{1}} \ldots L_{\bar{n}}\right) \operatorname{det}_{R} T \\
& =\left(q \gamma^{-n}\right)^{n-1} q a_{n} \operatorname{det}_{R} T,
\end{aligned}
$$

and so, under conditions $\operatorname{det}_{R} T=1, a_{n}=q^{-1} 1, \gamma^{n}=q$ we have $\operatorname{det}_{R}(L T)=1$. Here in the second line we substituted $A^{(n)} Z_{n}=q^{-n(n-1)} A^{(n)}$ and used the condition $\operatorname{rk} A^{(n)}=1$; in the last line we applied (2.4.5) and the definitions of $\operatorname{det}_{R} T$ and $a_{n}$.

In what follows we will investigate the automorphisms (4.1.1) for HD algebras of the types $\mathfrak{D} G L_{q}(n)[R, \gamma]$ and $\mathfrak{D} S L_{q}(n)[R]$. A key point for their dynamical interpretation is the possibility to write down the following ansatz:

$$
T(k+1)=L T(k)=\left(q a_{n}\right)^{1 / n} \Theta T(k) \Theta^{-1}, \text { where } \Theta \in \overline{\mathfrak{C h}}[R] .
$$

Here the dynamical process - evolution - is thought of as an inner HD algebra automorphism, and $\Theta$ plays a role of the evolution operator. As the evolution keeps $L$ unchanged, it is natural to assume that $\Theta$ belongs to the center of the RE algebra generated by the matrix $L$. More specifically, we will look for $\Theta$ as a formal power series in spectral variables $\mu_{\alpha}, \alpha=1, \ldots, n$, which we denote as $\overline{\mathfrak{C h}}[R]$. We also note that the condition 
$\Theta \in \overline{\mathfrak{C h}}[R]$ makes the ansatz manifestly covariant with respect to both left and right coactions (3.3.2), (3.3.3).

Factor $\left(q a_{n}\right)^{1 / n}$ in the ansatz (4.1.3) becomes trivial for the $S L_{q}(n)$ type HD algebra. In the $G L_{q}(n)$ case one adds this scaling factor to make the ansatz consistent with the evolution of $\operatorname{det}_{R} T$, see (4.1.2). One assumes the following relation for the newly introduced element $a_{n}^{1 / n}$ (cf. with (3.3.16)):

$$
T a_{n}^{1 / n}=\left(q \gamma^{-n}\right)^{2 / n} a_{n}^{1 / n} T \text {. }
$$

Then, consistency of (4.1.3) and (4.1.2) results in commutativity of $\operatorname{det}_{R} T$ with $\Theta$ :

$$
\operatorname{det}_{R} T \Theta=\Theta \operatorname{det}_{R} T,
$$

which again trivializes in the $S L_{q}(n)$ case.

Remark 4.2. The action of the automorphisms $\theta^{k}$ on $T$ can be equally treated as multiplications by powers of the left invariant matrix $M$ :

$$
T(k+1)=T(k) M=T M^{k}, \quad M(k)=M .
$$

The relation (3.4.1) between $L$ and $M$ would no more be valid if one would treat them as quantized right and left invariant Lie derivatives acting on the quantized external algebra of differential forms over the matrix group. In this case one would have a two-parametric series of automorphisms:

$$
\{T, L, M\} \stackrel{\theta^{(k, m)}}{\longrightarrow}\left\{L^{k} T M^{m}, L, M\right\}, \quad \forall k \geq 0, m \geq 0 .
$$

Example 4.3. Let us show that in the ribbon Hopf algebra setting the ribbon element $v \in \mathfrak{A}_{\mathcal{R}}$ generates the evolution (4.1.3) in the smash product algebra $\mathfrak{A}_{\mathcal{R}} \sharp \mathfrak{A}_{\mathcal{R}}^{*}$. For this we first have to specify pairing for the ribbon element. Using Definition (2.1.6) and relations (2.2.12), (2.4.3) and setting $\eta=q^{1 / n}$ as in Example 2.10 we calculate

$$
\left\langle T, v^{2}\right\rangle=\langle T, u S(u)\rangle=\rho_{V}(u) \rho_{V}(S(u))=\eta^{2} D_{R} C_{R}=q^{2\left(\frac{1}{n}-n\right)} I .
$$

Therefore, taking into account centrality of the ribbon element $v$ in $\mathfrak{A}_{\mathcal{R}}$, it is natural to define

$$
\langle T, v\rangle=q^{\left(\frac{1}{n}-n\right)} I
$$

Using this formula and relations (2.1.6), (3.2.5), (3.3.4) we now calculate conjugation of the matrix $T$ with the ribbon element

$$
\begin{aligned}
v T v^{-1} & =(v \otimes i d)\left\langle i d \otimes T, \Delta\left(v^{-1}\right)\right\rangle T=(v \otimes i d)\left\langle i d \otimes T,\left(v^{-1} \otimes v^{-1}\right) \mathcal{R}_{21} \mathcal{R}_{12}\right\rangle T \\
& =\left\langle T, v^{-1}\right\rangle\left\langle i d \otimes T, \mathcal{R}_{21} \mathcal{R}_{12}\right\rangle T=L T .
\end{aligned}
$$

Note that the defining relations for the evolution operator $\Theta$ (as a function of the spectral variables $\mu_{\alpha}$ ) and for the ribbon element $v$ both admit multiple solutions. ${ }^{9}$ Therefore, the problem of finding an explicit expression of the ribbon element $v$ in terms of spectral variables $\mu_{\alpha}$ demands further investigations.

\footnotetext{
9 The ribbon element is defined modulo the central factor $z \in \mathfrak{A}_{\mathcal{R}}: z^{2}=1, S(z)=z, \epsilon(z)=1, \Delta(z)=$ $z \otimes z$. For the evolution operator $\Theta(\mu)$ different solutions are constructed in the next sections.
} 
4.2. Equations for the evolution operator $\Theta$. Using the results of Sect. 3 it is straightforward to derive equations for $\Theta$. We consider in detail, the evolution in the $S L_{q}(n)$ type HD algebra. In this case we assume

$$
\Theta=\Theta\left(\mu_{1}, \ldots, \mu_{n}\right), \quad \text { where } a_{n}=\prod_{\alpha=1}^{n} \mu_{\alpha}=q^{-1} \text { and } \gamma=q^{-1 / n} .
$$

Applying from the left the projector $P^{\alpha}$ to both sides of (4.1.3) we obtain

$$
q \mu_{\alpha}\left(P^{\alpha} T\right)=\Theta\left(P^{\alpha} T\right) \Theta^{-1}, \quad \forall \alpha=1, \ldots, n .
$$

Multiplying this equality by $\Theta$ from the right and permuting $\Theta$ with $P^{\alpha} T$ in the left-hand side with the help of (3.3.17) we finally get

$$
q \mu_{\alpha} \Theta\left(q^{-2 / n} \mu_{1}, \ldots, q^{2-2 / n} \mu_{\alpha}, \ldots, q^{-2 / n} \mu_{n}\right)\left(P^{\alpha} T\right)=\Theta\left(\mu_{1}, \ldots, \mu_{n}\right)\left(P^{\alpha} T\right) .
$$

We state the result in the following proposition:

Proposition 4.4. For the Heisenberg double algebra $\mathfrak{D} S L_{q}(n)[R]$ the evolution operator $\Theta\left(\mu_{\alpha}\right)$ in (4.1.3), (4.2.1) is a solution of equations

$$
q \mu_{\alpha} \Theta\left(\nabla^{\alpha}\left(\mu_{\beta}\right)\right)=\Theta\left(\mu_{\beta}\right) \quad \forall \alpha=1, \ldots, n,
$$

where $\nabla^{\alpha}$ are finite shift operators introduced in (3.5.13). In the $S L_{q}(n)$ case their actions are

$$
\nabla^{\alpha}\left(\mu_{\beta}\right):=q^{2 X_{\alpha \beta}} \mu_{\beta}, \quad X_{\alpha \beta}:=\delta_{\alpha \beta}-\frac{1}{n} \quad \forall \alpha, \beta=1, \ldots, n .
$$

The $n \times n$ matrix $X$ is a Gram matrix for the set of vectors $\vec{e}_{\alpha}^{*} \in \mathbb{Q}^{n}, \alpha=1, \ldots, n$ :

$$
\vec{e}_{\alpha}^{*}:=\frac{1}{n}(\underbrace{-1, \ldots,-1}_{(\alpha-1) \text { times }}, n-1,-1, \ldots,-1), \quad X_{\alpha \beta}=\left\langle\vec{e}_{\alpha}^{*}, \vec{e}_{\beta}^{*}\right\rangle .
$$

$X$ is positive semi-definite of rank $n-1\left(\sum_{\alpha=1}^{n} \vec{e}_{\alpha}^{*}=0\right)$.

For the Heisenberg double algebra $\mathfrak{D G}_{G}(n)[R, \gamma]$ the evolution operator $\Theta$ is suitably parameterized by variables $z:=\left(q a_{n}\right)^{1 / n}$ and $v_{\alpha}$,

$v_{\alpha}:=\mu_{\alpha}\left(q a_{n}\right)^{-1 / n}, \quad$ such that $\quad \prod_{\alpha=1}^{n} v_{\alpha}=q^{-1}, \quad v_{\alpha} \operatorname{det}_{R} T=\operatorname{det}_{R} T v_{\alpha} \forall \alpha$.

The evolution equations for $\Theta\left(v_{1}, \ldots, v_{n} ; z\right)$ read

$$
q v_{\alpha} \Theta\left(\nabla^{\alpha}\left(v_{\beta}\right) ;\left(q \gamma^{-n}\right)^{2 / n} z\right)=\Theta\left(v_{\alpha} ; z\right) \forall \alpha=1, \ldots, n,
$$

where shift operators $\nabla^{\alpha}$ are defined as in (4.2.3). Since $\prod_{\alpha=1}^{n} \nabla^{\alpha}=1$, this system is consistent provided that $\Theta\left(v_{\beta} ; q^{2} \gamma^{-2 n} z\right)=\Theta\left(v_{\beta} ; z\right)$ (cf. with (4.1.5)). Demanding that $\Theta$ does not actually depend on zone reduces (4.2.4) to (4.2.2).

Proof. The $S L_{q}(n)$ case is already considered. Taking into account relations (4.1.4) and (3.3.19) a derivation of the evolution equations in the $G L_{q}(n)$ case is the same.

In the next two subsections we will construct particular solutions of the $S L$-type evolution equations (4.2.2). 
4.3. Solution in case $|q|<1$. Let us look for the solution of (4.2.2) as a series in $\mu_{\alpha}$. Taking into account condition (4.2.1) we exclude one dependent variable, say $\mu_{n}$, from the expansion

$$
\Theta\left(\mu_{\alpha}\right)=\sum_{\vec{k} \in \mathbb{Z}^{n-1}} c(\vec{k}) \mu_{1}^{k_{1}} \mu_{2}^{k_{2}} \ldots \mu_{n-1}^{k_{n-1}},
$$

where $\mathbb{Z}^{n-1}=\left\{\left(k_{1}, \ldots, k_{n-1}\right): k_{i} \in \mathbb{Z}\right\}$, and the coefficients $c(\vec{k})$ are $\mathbb{C}$-valued functions on $\mathbb{Z}^{n-1}$. Substitution of (4.3.1) into (4.2.2) gives conditions on the coefficients:

$$
c\left(\vec{k}+\vec{\epsilon}_{\alpha}\right)=q^{\left(1+\frac{2}{n} \sum_{\beta=1}^{n-1} A_{\alpha \beta}^{*} k_{\beta}\right)} c(\vec{k}) \quad \forall \alpha=1, \ldots, n-1 .
$$

Here $\vec{\epsilon}_{\alpha}:=(\underbrace{0, \ldots, 0}_{(\alpha-1) \text { times }}, 1,0, \ldots, 0)$, and $A_{\alpha \beta}^{*}:=n X_{\alpha \beta}$ is a $(n-1) \times(n-1)$ positivedefinite matrix.

The general solution to (4.3.2) is

$$
c(\vec{k})=q^{\frac{1}{n}\left\{\left(\vec{k}, A^{*} \vec{k}\right)+(\overrightarrow{1} \vec{k})\right\}},
$$

where we choose normalization $c(\overrightarrow{0})=1$ and use notation $\left(\vec{k}, A^{*} \vec{k}\right)=\sum_{\alpha, \beta=1}^{n-1} k_{\alpha} A_{\alpha \beta}^{*}$ $k_{\beta}$, and $\overrightarrow{1}=(1, \ldots, 1)$, so that $(\overrightarrow{1}, \vec{k})=\sum_{\alpha=1}^{n-1} k_{\alpha}$.

Remark 4.5. The matrix $A_{\alpha \beta}^{*}$ is a Gram matrix of a lattice $A_{n-1}^{*}$ dual to the root lattice $A_{n-1}$ (see [CS], Chap. 4, Sect. 6.6). The corresponding quadratic form $\left(\vec{k}, A^{*} \vec{k}\right)$ is often referred to as Voronoi's principal form of the first type.

The ansatz (4.3.1) gives a particular solution (4.3.3) of the evolution equations; we denote it $\Theta^{(1)}$. Introducing a parameterization,

$$
\begin{aligned}
& q=\exp (2 \pi \mathrm{i} \tau), \quad q^{1 / n} \mu_{\alpha}=\exp \left(2 \pi \mathrm{i} z_{\alpha}\right): \\
& \sum_{\alpha=1}^{n-1} z_{\alpha}=0, \Omega_{\alpha \beta}=\frac{2 \tau}{n} A_{\alpha \beta}^{*}=2 \tau\left(\delta_{\alpha \beta}-\frac{1}{n}\right),
\end{aligned}
$$

we can write $\Theta^{(1)}$ as a Riemann theta function $\theta(\vec{z}, \Omega)$ (see [Mum])

$$
\Theta^{(1)}\left(\mu_{\alpha}\right)=\theta(\vec{z}, \Omega)=\sum_{\vec{k} \in \mathbb{Z}^{n-1}} \exp \{\pi \mathrm{i}(\vec{k}, \Omega \vec{k})+2 \pi \mathrm{i}(\vec{k}, \vec{z})\} .
$$

Here $\tau$ is a modular parameter and $\Omega$ is a matrix of periods. Expression (4.3.5) converges either if $|q|<1$, or if $q$ is a rational root of unity, in which case the series can be truncated.

Remark 4.6. One can present formula (4.3.5) in a manifestly covariant form:

$$
\Theta^{(1)} \equiv \Theta^{(1)}\left(\vec{z}, A_{n-1}^{*}, \tau\right)=\sum_{\overrightarrow{\mathrm{k}} \in A_{n-1}^{*}} \exp \left\{2 \pi \mathrm{i} \frac{\tau}{n}\langle\overrightarrow{\mathrm{k}}, \overrightarrow{\mathrm{k}}\rangle+2 \pi \mathrm{i}\langle\overrightarrow{\mathrm{k}}, \overrightarrow{\mathrm{z}}\rangle\right\} .
$$

Here vectors $\overrightarrow{\mathrm{k}}=\sum_{\alpha=1}^{n-1} k_{\alpha} e_{\alpha}^{*}$ label vertices of the lattice $A_{n-1}^{*}$, and $\overrightarrow{\mathrm{z}}=\sum_{\alpha=1}^{n-1} z_{\alpha} e_{\alpha}$, where $e_{\alpha}=\epsilon_{\alpha}-\epsilon_{n}, \alpha=1, \ldots, n-1$, (see the line below (4.3.2)) are basic vectors of the root lattice $A_{n-1}:\left\langle e_{\alpha}^{*}, e_{\beta}\right\rangle=\delta_{\alpha \beta}$. 
In the simplest $S L_{q}(2)$ case the evolution operator $\Theta^{(1)}$ becomes the Jacobi theta function:

$$
\Theta^{(1)}\left(\mu_{1}\right)=\sum_{k \in \mathbb{Z}} q^{\frac{1}{2} k(k+1)} \mu_{1}^{k}=\sum_{k \in \mathbb{Z}} \exp \left(\pi \mathrm{i} k^{2} \tau+2 \pi \mathrm{i} k z_{1}\right)=\theta_{3}\left(z_{1} ; q\right),
$$

or, in a multiplicative form

$$
\Theta^{(1)}\left(\mu_{1}\right)=\prod_{n=1}^{\infty}\left(1-q^{n}\right)\left(1+q^{n} \mu_{1}\right)\left(1+q^{n-1} / \mu_{1}\right) .
$$

4.4. Solution for arbitrary $q$. In this section we derive yet another particular solution of the evolution equations (4.2.2), the one which is well defined for arbitrary values of $q$. The idea of such a solution was proposed in L.D. Faddeev's lectures on twodimensional integrable quantum field theory [F.94] (see also [F.95]). We use heuristic arguments inspired by considerations in [AF.91]. For the moment we assume $\operatorname{dim} V=n$, so that the range of the indices $\alpha$ and $i, j$ in the projectors $P_{i j}^{\alpha}, S_{i j}^{\alpha}$ is the same. Consider the following $n \times n$ matrices:

$$
U_{i j}:=\sum_{k=1}^{n} u_{i k} P_{k j}^{\alpha=i}, \quad V_{i j}:=\sum_{k=1}^{n} S_{i k}^{\alpha=j} v_{k j},
$$

where the only restriction for the auxiliary parameters $u_{i j}$ and $v_{i j}$ is their commutativity with the spectral variables $\mu_{\alpha}$,

$$
\left[u_{i j}, \mu_{\alpha}\right]=\left[v_{i j}, \mu_{\alpha}\right]=0 \quad \forall i, j, \alpha .
$$

As a result of the Cayley-Hamilton identities (3.2.24), (3.4.11) we have matrix relations

$$
U L=q D U, \quad M V=\gamma^{2} q^{-1} V D, \quad \text { where } \quad D:=\operatorname{diag}\left\{\mu_{1}, \ldots, \mu_{n}\right\} .
$$

Moreover, by (3.4.13), matrix $Q:=U T V$ is diagonal

$$
Q=\operatorname{diag}\left\{w_{1}, \ldots, w_{n}\right\}, \quad \text { where } w_{i}:=\left(u P^{i} T v\right)_{i i},
$$

and, by (3.3.17), $w_{i}$ satisfy the following permutation relations with $\mu_{j}$ and $z_{j}$ :

$$
w_{i} \mu_{j}=q^{2 \delta_{i j}} \gamma^{-2} \mu_{j} w_{i} \Leftrightarrow w_{i} z_{j}=\left(z_{j}+2 \tau\left(\delta_{i j}-1 / n\right)\right) w_{i},
$$

where in the latter formula we used the $S L_{q}(n)$ type condition $\gamma=q^{1 / n}$.

Assuming invertibility of the matrices $U$ and $V$ we can write diagonal decompositions for the matrices $L, M$ and $T$,

$$
L=q U D U^{-1}, \quad M=\gamma^{2} q^{-1} V D V^{-1}, \quad T=U^{-1} Q V^{-1},
$$

which after substitution into the ansatz (4.1.3) reduce the evolution equations to the following form:

$$
q D Q=\Theta Q \Theta^{-1} \quad \Leftrightarrow \quad q \mu_{i} w_{i}=\Theta w_{i} \Theta^{-1} .
$$


Taking into account (4.4.1) these equations clearly have the following solution:

$$
\Theta^{(2)}\left(z_{\alpha}\right):=\exp \left(-\frac{\pi \mathrm{i}}{2 \tau} \sum_{\beta=1}^{n} z_{\beta}^{2}\right)
$$

Now, it is easy to check that the function $\Theta^{(2)}$ fulfills the evolution equations (4.2.2) without additional assumptions we made for the derivation. Written in the independent variables $\vec{z}=\left\{z_{1}, \ldots, z_{p-1}\right\}$ it reads

$$
\Theta^{(2)}(\vec{z})=\exp \left(-\frac{\pi \mathrm{i}}{\tau} \sum_{1 \leq \alpha \leq \beta \leq n-1} z_{\alpha} z_{\beta}\right)=\exp \left\{-\pi \mathrm{i}\left(\vec{z}, \Omega^{-1} \vec{z}\right)\right\},
$$

where the inverse matrix of periods is

$$
\Omega_{\alpha \beta}^{-1}=\frac{1}{2 \tau}\left(\delta_{\alpha \beta}+1\right)=\frac{1}{2 \tau} A_{\alpha \beta}
$$

and $A_{\alpha \beta}=\left\langle e_{\alpha}, e_{\beta}\right\rangle$ is the Gram matrix for the root lattice $A_{n-1}$ (see Remark 4.6). Let us stress that the logarithmic change of variables $\mu_{\alpha} \mapsto z_{\alpha}$ (4.3.4) which was rather superficial in the case of $\Theta^{(1)}$, is inevitable for the derivation of $\Theta^{(2)}$.

Finally, we comment on the relation between the two evolution operators $\Theta^{(1)}=$ $\theta(\vec{z}, \Omega)$ and $\Theta^{(2)}$. The relation is based on a functional equation for the Riemann theta function:

$$
\theta\left(\Omega^{-1} \vec{z},-\Omega^{-1}\right)=(\operatorname{det}(\Omega / \mathrm{i}))^{\frac{1}{2}} \exp \left\{\pi \mathrm{i}\left(\vec{z}, \Omega^{-1} \vec{z}\right)\right\} \theta(\vec{z}, \Omega)
$$

which is the special case of a more general modular functional equation (for derivation and generalization see [Mum], Chap. 2, Sect. 5). With our particular matrix of periods $\Omega(4.3 .5)$ we find

$$
\Theta^{(2)}(\vec{z})=\frac{1}{\sqrt{n}}\left(\frac{2 \tau}{\mathrm{i}}\right)^{\frac{n-1}{2}} \frac{\theta(\vec{z}, \Omega)}{\theta\left(\Omega^{-1} \vec{z},-\Omega^{-1}\right)} .
$$

Note that the theta function in the denominator $-\theta\left(\Omega^{-1} \vec{z},-\Omega^{-1}\right)-$ commutes with the elements of $\mathfrak{D} S L_{q}(n)[R]$ and can be thought as an evolution operator on a 'modular dual' quantum cotangent bundle [F.99].

\section{Appendix A. Pairing Between Spectral Variables and Quantized Functions}

Here we calculate pairing of the elementary symmetric functions $a_{i}$ (3.2.17) with the generators of quantized functions $T_{j}^{i}$. We assume that $T$ and $a_{i}$ are realized respectively, as elements of dual quasi-triangular Hopf algebras $\mathfrak{A}_{\mathcal{R}}^{*}$ and $\mathfrak{A}_{\mathcal{R}}$. We further extend this pairing also for the spectral variables $\mu_{\alpha}$. 
For the calculation we use formula

$$
\left\langle T_{1}, L_{2}\right\rangle=\eta^{-2} q^{\left(n-\frac{1}{n}\right)} R_{12}^{2},
$$

which follows from the definitions (2.2.11), (3.1.5), (3.2.3), (3.2.5).

Proposition A.1. Let $a_{i}$ (3.2.3), (3.2.5) and $T$ (3.1.5) be elements of the dual quasi-triangular Hopf algebras, respectively, $\mathfrak{A}_{\mathcal{R}}$ and $\mathfrak{A}_{\mathcal{R}}^{*}$. Assume that the $R$-matrix $R(2.2 .11)$ is $G L_{q}(n)$ type with scaling parameter $\eta=q^{1 / n}$ as in Example 2.10. Then

$$
\left\langle T, a_{i}\right\rangle=q^{-3 i / n} n_{q}^{-1}\left(\begin{array}{l}
n \\
i
\end{array}\right)_{q}\left\{n_{q}+q^{n+1}-q^{n-2 i+1}\right\} I .
$$

Proof. The calculation proceeds as follows:

$$
\begin{aligned}
\left\langle T_{1}, a_{i}\right\rangle= & \left\langle T_{1}, \operatorname{Tr}_{R}(2, \ldots, i+1)\left(A^{(i)} L_{1} \ldots L_{i}\right)^{\uparrow 1}\right\rangle \\
= & q^{i\left(n-\frac{3}{n}\right)} \operatorname{Tr}_{R}(2, \ldots, i+1)\left(A^{(i) \uparrow 1}\left(J_{2} \ldots J_{i+1}\right)\left(J_{1}^{-1} \ldots J_{i}^{-1}\right)^{\uparrow 1}\right) \\
= & q^{i\left(n-\frac{3}{n}\right)} \operatorname{Tr}_{R}(2, \ldots, i+1)\left(R_{1} \ldots R_{i} A^{(i)} R_{i} \ldots R_{1}\right) \\
= & q^{i\left(n-\frac{3}{n}\right)}\left\{q^{-n}(n+1-i)_{q} i_{q}^{-1} \operatorname{Tr}_{R}(2, \ldots, i)\right. \\
& +\left(R_{1} \ldots R_{i-1} A^{(i-1)} R_{i-1} \ldots R_{1}\right) \\
\ldots= & \left.q^{i\left(n-\frac{3}{n}\right)}\left(\begin{array}{c}
n \\
i
\end{array}\right) q\left\{q^{-1}\right) q^{-(n+2)(i-1)} n_{q}^{-1}\left(\begin{array}{c}
n \\
i
\end{array}\right)_{q} I_{1}\right\}
\end{aligned}
$$

Here in the first line we substituted an expression for $a_{i}$ similar to (3.3.13). In the second line we evaluated the pairing using formulas $\left\langle T_{1},\left(L_{\bar{i}}\right)^{\uparrow 1}\right\rangle=\eta^{-2} q^{\left(n-\frac{1}{n}\right)} J_{i+1}\left(J_{i}^{-1}\right)^{\uparrow 1}$ following from (A.1). In the third line we first, used the cyclic property of the R-trace to evaluate the term $\left(J_{1}^{-1} \ldots J_{i}^{-1}\right)^{\uparrow 1}$ on $\left(A^{(i)}\right)^{\uparrow 1}$, and then rearranged the product $J_{2} \ldots J_{i+1}=$ $Z_{i+1}=J_{2}^{\dagger} \ldots J_{i+1}^{\dagger}$, where $J_{1}^{\dagger}:=I, \quad J_{k+1}^{\dagger}:=R_{i-k+1} J_{k}^{\dagger} R_{i-k+1}$, and evaluated the term $J_{2}^{\dagger} \ldots J_{i}^{\dagger}$ on $\left(A^{(i)}\right)^{\uparrow 1}$. After that we recollected terms in the product: $\left(A^{(i)}\right)^{\uparrow 1} J_{i+1}^{\dagger}=$ $R_{1} \ldots R_{i} A^{(i)} R_{i} \ldots R_{1}$. In the fourth line we substituted $R_{i}=R_{i}^{-1}+\left(q-q^{-1}\right) I$ for one of $R_{i} \mathrm{~s}$ and used formulas (2.2.8) and (2.2.10) to evaluate $\operatorname{Tr}_{R}(i+1)$. Then, in the summand which is proportional to $\left(q-q^{-1}\right)$ all the R-traces can be evaluated with the help of (2.4.4). Omission points in the fifth line stand for similar evaluations of $\operatorname{Tr}_{R}(i) \ldots \operatorname{Tr}_{R}(2)$; the resulting expression coincides with (A.2).

For $a_{n}$ relation (A.2) simplifies to

$$
\left\langle T, a_{n}\right\rangle=q^{-1} I,
$$

which obviously agrees with (3.2.18). So, we checked a consistency of the normalizations $q^{n-\frac{1}{n}}$ in (3.2.5) and $\eta=q^{1 / n}$ for the Drinfeld-Jimbo R-matrices with the $S L_{q}(n)$ reduction condition (3.2.18).

Corollary A.2. In the conditions of Proposition A.1 the pairing $\langle\cdot, \cdot\rangle$ can be extended for the spectral variables (3.2.22):

$$
\left\langle T, \mu_{\alpha}\right\rangle=q^{\left(2 \alpha+2 \delta_{\alpha n}-n-\frac{3}{n}-1\right)} I, \quad \alpha=1, \ldots, n .
$$


Proof. Let us rescale the spectral variables $\tilde{\mu}_{\alpha}:=q^{\frac{3}{n}-2 \delta_{\alpha n}} \mu_{\alpha}$. For rescaled variables (A.4) reads

$$
\left\langle T, \tilde{\mu}_{\alpha}\right\rangle=q^{(2 \alpha-n-1)} I .
$$

Using the q-binomial identity $q^{i}\left(\begin{array}{c}n-1 \\ i\end{array}\right)_{q}+q^{i-n}\left(\begin{array}{c}n-1 \\ i-1\end{array}\right)_{q}=\left(\begin{array}{c}n \\ i\end{array}\right)_{q}$, it is straightforward to derive from (A.5) pairings of the elementary symmetric functions $e_{i}(\tilde{\mu})$ by induction on $n:\left\langle T, e_{i}(\tilde{\mu})\right\rangle=\left(\begin{array}{l}n \\ i\end{array}\right)_{q}$. Using (3.3.18) it is then straightforward to derive pairings for elementary symmetric functions in original spectral variables $-\left\langle T, e_{i}(\mu)\right\rangle-$ which under the identification $a_{i} \mapsto e_{i}(\mu)$ coincide with (A.2).

\section{Appendix B. Three Lemmas for Subsection 3.4}

Here we collect some technical results which are used for establishing the relation between the spectra of the left and right invariant vector fields.

Lemma B.1. a) If the R-matrix $R$ is skew invertible then the following four statements are equivalent: i) the matrix $D_{R}$ is invertible; ii) the matrix $C_{R}$ is invertible; iii) the R-matrix $R^{-1}$ is skew invertible; iv) the $R$-matrix $\stackrel{*}{R}:=P R^{-1} P$ is skew invertible. One has

$$
D_{R}^{*}=C_{R^{-1}}=\left(D_{R}\right)^{-1}, \quad C_{R}=D_{R^{-1}}=\left(C_{R}\right)^{-1} .
$$

b) Let $R$ be the Hecke type R-matrix generating representations $\rho_{R}(2.3 .7)$ of the algebras $\mathcal{H}_{k}(q)$. Then the $\mathrm{R}$-matrix $\stackrel{*}{R}$ is Hecke type as well, and $\rho_{R}^{*}$ are representations of the algebras $\mathcal{H}_{k}\left(q^{-1}\right)$. If additionally the parameter $q$ satisfies conditions [k] (2.3.1) so that the idempotent $\left.a^{(k)}\right|_{q \leftrightarrow q^{-1}} \in \mathcal{H}_{k}\left(q^{-1}\right)$, (see (2.3.2)) is well defined, then

$$
\begin{aligned}
& \stackrel{*}{A^{(k)}}:=\rho_{R}\left(\left.a^{(k)}\right|_{q \leftrightarrow q^{-1}}\right)=\Upsilon_{P}^{(k)} A^{(k)} \Upsilon_{P}^{(k)}, \\
R_{i} & \stackrel{*}{A^{(k)}}=-q \stackrel{*}{A^{(k)}}, \quad \forall i=1, \ldots, k-1 .
\end{aligned}
$$

Here $\Upsilon_{P}^{(k)}=\left(\Upsilon_{P}^{(k)}\right)^{-1}$ is a particular $R=P$ case of an operator $\Upsilon_{R}^{(k)} \in \operatorname{Aut}\left(V^{\otimes k}\right)$, defined inductively for any R-matrix $R$,

$$
\Upsilon_{R}^{(1)}:=1, \quad \Upsilon_{R}^{(k+1)}:=\left(R_{1} R_{2} \ldots R_{k}\right) \Upsilon_{R}^{(k)}=\Upsilon_{R}^{(k)}\left(R_{k} \ldots R_{2} R_{1}\right) \quad \forall k=2,3, \ldots
$$

This operator performs reflection of the indices of the R-matrices,

$$
R_{i} \Upsilon_{R}^{(k)}=\Upsilon_{R}^{(k)} R_{k-i} \quad \forall i, k: 1 \leq i<k .
$$

The particular element $\Upsilon_{P}^{(k)}$ enjoys also relations

$$
\begin{gathered}
R_{i} \Upsilon_{P}^{(k)}=\Upsilon_{P}^{(k)}(P R P)_{k-i} \quad \forall i, k: 1 \leq i<k, \quad \forall \text { R-matrix } R, \\
M_{i} \Upsilon_{P}^{(k)}=\Upsilon_{P}^{(k)} M_{k-i+1} \quad \forall i, k: 1 \leq i \leq k, \quad \forall M \in \operatorname{End}_{W}(V),
\end{gathered}
$$

where $W$ is an arbitrary $\mathbb{C}$-linear space. 
Proof. The first equality in both formulas (B.1) is proved in a more general setting in [OP.05], Lemma 3.6 c). The second equality is proved in [Is.04], Sect. 3.1, Proposition 2. Relations (B.5) and (B.6) for matrices $\Upsilon_{R}^{(k)}, \Upsilon_{P}^{(k)}$ follow directly from (2.2.4) and from equalities

$$
R_{1} P_{2} P_{1}=P_{2} P_{1} R_{2}, \quad R_{2} P_{1} P_{2}=P_{1} P_{2} R_{1} \text {. }
$$

Equalities (B.7) are obvious. Relations (B.3) are byproducts of (B.2) and (2.3.4). The second equality in (B.2) follows from (2.3.2), (2.3.3), (B.6), and the Hecke relation for $\stackrel{*}{R}: \stackrel{*}{R}=P R P-\left(q-q^{-1}\right) I$.

Lemma B.2. Let $M$ be a matrix of left invariant vector fields for the Hecke type HD algebra $\mathfrak{D G}[R, \gamma]$. For any $i \geq 1$ and $j \geq 0$ one has

$$
\begin{aligned}
& \left(M_{\frac{*}{1}} \stackrel{*}{J}_{1}\right)\left(M_{\frac{*}{2}} \vec{J}_{2}^{*}\right) \ldots\left(M_{\frac{*}{i}}^{*} J_{i}^{*}\right) I_{i+1, \ldots, i+j} \\
& =\gamma^{i(i+1)} \operatorname{Tr}_{R}(i+j+1, \ldots, 2 i+j) \\
& \left(\Upsilon_{P}^{(i+j)} \Upsilon_{P}^{(2 i+j)}(L T)_{i} \ldots(L T)_{1} \Upsilon_{R}^{(2 i)}\left(T_{i} \ldots T_{1}\right)^{-1} \Upsilon_{P}^{(2 i+j)} \Upsilon_{P}^{(i+j)}\right),
\end{aligned}
$$

where $I_{i+1, \ldots, i+j}$ is the identity operator acting in the component spaces $V$ with labels $i+1, \ldots, i+j$.

Proof. Consider the following sequence of transformations:

$$
\begin{aligned}
& M_{\frac{*}{1}} \stackrel{*}{J}_{1}=M_{1}=\left(T^{-1} L T\right)_{1}=\operatorname{Tr}_{R}(2)\left(\underline{T_{1}^{-1} R_{1} L_{1}} T_{1}\right)=\gamma^{2} \operatorname{Tr}_{R}^{(2)}\left(L_{2} \underline{T_{1}^{-1} R^{-1} T_{1}}\right) \\
& =\gamma^{2} \operatorname{Tr}_{R}(2)\left((L T)_{2} R_{1}^{-1} T_{2}^{-1}\right)=\gamma^{2} \operatorname{Tr}_{R}(2)\left(P_{1}(L T)_{1} \stackrel{*}{R}_{1} T_{1}^{-1} P_{1}\right) .
\end{aligned}
$$

Here in the first line we transform the underlined expressions using (3.3.1) and (3.1.1), and in the last line we apply the definition (3.4.5). Relation (B.10) reproduces formula (B.9) for $i=1$ and $j=0$. By a repeated application of formula (cf. with (2.2.10))

$$
\operatorname{Tr}_{R}(j+1)\left(P_{j} X P_{j}\right)=I_{j} \operatorname{Tr}_{R}(j)(X) \quad \forall X \in \operatorname{End}_{W}\left(V^{\otimes j}\right),
$$

we can rewrite it as (B.9) with $i=1$ and arbitrary $j>0$,

$$
\left(M_{\underline{*}} \stackrel{*}{J}_{1}\right) I_{2, \ldots j+1}=\gamma^{2} \operatorname{Tr}_{R}(j+2)\left(\left(P_{j+1} \ldots P_{1}\right)(L T)_{1} \stackrel{*}{R}_{1} T_{1}^{-1}\left(P_{1} \ldots P_{j+1}\right)\right) .
$$

In a similar way, for any value of $i$ relations, (B.9) with $j>0$ follow from that with $j=0$ by a repeated application of (B.11). Therefore, it is enough to consider the case $j=0$.

Using relations (B.12) and (B.8) we can rewrite an expression $M_{\frac{*}{i}} \stackrel{*}{J}$ in the following way 


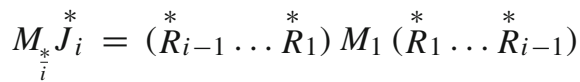

$$
\begin{aligned}
& =\gamma^{2} \operatorname{Tr}_{R}(i+1)\left(\left(P_{i} \ldots P_{2} P_{1}\right)(L T)_{1}\left(\stackrel{*}{R}_{i} \ldots \stackrel{*}{R_{2}} \stackrel{*}{R}_{1} \stackrel{*}{R}_{2} \ldots \stackrel{*}{R}_{i}\right)\left(T_{1}\right)^{-1}\left(P_{1} P_{2} \ldots P_{i}\right)\right) .
\end{aligned}
$$

Now we are ready to prove formula (B.9) by induction on $i$. Assuming that (B.9) with $j=1$ is valid for the product of $(i-1)$ factors we transform the product of $i$ factors,

$$
\begin{aligned}
& \left(M_{\frac{*}{1}} \stackrel{*}{J}_{1}\right)\left(M_{\frac{*}{2}} \stackrel{*}{J}_{2}\right) \ldots\left(M_{\frac{*}{i}} \stackrel{*}{J}_{i}\right) \\
& =\gamma^{(i-1) i} \operatorname{Tr}_{R}(i+1, \ldots 2 i-1)\left(\Upsilon_{P}^{(i)} \Upsilon_{P}^{(2 i-1)}(L T)_{i-1} \ldots(L T)_{1}\right. \\
& \left.\times \Upsilon_{R}^{(2 i-2)}\left(T_{i-1} \ldots T_{1}\right)^{-1} \Upsilon_{P}^{(2 i-1)} \Upsilon_{P}^{(i)}\right)\left(M_{\frac{*}{i} J_{i}^{*}}^{*}\right)
\end{aligned}
$$

Next, we apply formulas (B.6), (B.7) to move the last factor $\left(M_{\frac{*}{i}}{ }_{i}^{*}\right)$ in this expression left-wards. The result is

$$
\begin{aligned}
= & \gamma^{(i-1) i} \operatorname{Tr}_{R}(i+1, \ldots 2 i-1) \\
& \times\left(\Upsilon_{P}^{(i)} \Upsilon_{P}^{(2 i-1)}(L T)_{i-1} \ldots(L T)_{1} \Upsilon_{*}^{(2 i-2)}\left(T_{i-2} \ldots T_{1}\right)^{-1}\right. \\
& \left.\left.\left(M_{\dot{*}} J_{i}^{*}\right)^{\uparrow 1}\right)^{\uparrow(i-2)} \Upsilon_{P}^{(2 i-1)} \Upsilon_{P}^{(i)}\right),
\end{aligned}
$$

where we have used identities $\left(T_{i-1}\right)^{-1}=\left(T_{1}^{-1}\right)^{\uparrow(i-2)}$ and $\left(M_{\frac{*}{i}}^{\stackrel{*}{J}}\right)^{\uparrow(i-1)}=$ $\left(\left(M_{\frac{*}{i}} \stackrel{*}{J}_{i}\right)^{\uparrow 1}\right)^{\uparrow(i-2)}$ to arrange the terms $\left(T_{i-1}\right)^{-1}$ and $\left(M_{\frac{*}{i}} \stackrel{*}{J}_{i}\right)^{\uparrow(i-1)}$ in a suitable way.

Next, we use formula (3.4.8) for their permutation and then, in a similar way we move term $\left(M_{*} \stackrel{*}{J}\right)$ to the left of all the terms $\left(T_{*}\right)^{-1}$ :

$$
\begin{aligned}
\cdots= & \gamma^{(i-1) i+2(i-1)} \operatorname{Tr}_{R}(i+1, \ldots 2 i-1)\left(\Upsilon_{P}^{(i)} \Upsilon_{P}^{(2 i-1)}(L T)_{i-1} \ldots(L T)_{1} \Upsilon_{R}^{(2 i-2)}\right. \\
& \left.\times\left(M_{\frac{*}{2 i-1}} J_{2 i-1}^{*}\right)\left(T_{i-1} \ldots T_{1}\right)^{-1} \Upsilon_{P}^{(2 i-1)} \Upsilon_{P}^{(i)}\right)
\end{aligned}
$$

Now we substitute the expression (B.13) for $\left(M_{\frac{*}{2 i-1}} J_{2 i-1}^{*}\right)$

$$
\begin{aligned}
= & \gamma^{i(i+1)} \operatorname{Tr}_{R}(i+1, \ldots 2 i)\left(\Upsilon_{P}^{(i)} \Upsilon_{P}^{(2 i-1)}(L T)_{i-1} \ldots(L T)_{1} \Upsilon_{R}^{(2 i-2)}\left(P_{2 i-1} \ldots P_{1}\right)(L T)_{1}\right. \\
& \left.\times\left(\stackrel{*}{R}_{2 i-1} \ldots \stackrel{*}{R}_{2} \stackrel{*}{R}_{1} \stackrel{*}{R}_{2} \ldots \stackrel{*}{R}_{2 i-1}\right)\left(T_{1}\right)^{-1}\left(P_{1} \ldots P_{2 i-1}\right)\left(T_{i-1} \ldots T_{1}\right)^{-1} \Upsilon_{P}^{(2 i-1)} \Upsilon_{P}^{(i)}\right)
\end{aligned}
$$


and move the term $\left(P_{2 i-1} \ldots P_{1}\right)$ leftwards and the term $\left(P_{1} \ldots P_{2 i-1}\right)$ rightwards close to the terms $\Upsilon_{P}^{(2 i-1)}$. Finally, using (B.4) we complete the calculation

$$
=\gamma^{i(i+1)} \operatorname{Tr}_{R}(i+1, \ldots 2 i)\left(\Upsilon_{P}^{(i)} \Upsilon_{P}^{(2 i)}(L T)_{i} \ldots(L T)_{1} \Upsilon_{*}^{(2 i)}\left(T_{i} \ldots T_{1}\right)^{-1} \Upsilon_{P}^{(2 i)} \Upsilon_{P}^{(i)}\right)
$$

Here we transformed terms containing $\stackrel{*}{R}$ in the following way:

$$
\begin{aligned}
& \Upsilon_{R}^{(2 i-2) \uparrow 1}\left(\stackrel{*}{R} 2 i-1 \ldots \stackrel{*}{R}_{2} \stackrel{*}{R}_{1} \stackrel{*}{R}_{2} \ldots \stackrel{*}{R}_{2 i-1}\right) \\
& =\Upsilon_{R}^{(2 i-2) \uparrow 1}\left(\stackrel{*}{R}{ }_{1} \ldots \stackrel{*}{R} 2 i-2 \stackrel{*}{R}_{2 i-1} \stackrel{*}{R} 2 i-2 \ldots \stackrel{*}{R} 1\right) \\
& =\left(\stackrel{*}{R}_{1} \ldots \stackrel{*}{R}_{2 i-1}\right) \Upsilon_{R}^{(2 i-2)}\left(\stackrel{*}{R}_{2 i-2} \ldots \stackrel{*}{R}_{1}\right)=\left(\stackrel{*}{R}_{1} \ldots \stackrel{*}{R}_{2 i-1}\right) \Upsilon_{R}^{(2 i-1)}=\Upsilon_{R}^{(2 i)} .
\end{aligned}
$$

Lemma B.3. The operators $J_{k}(3.3 .8)$ and $\Upsilon^{(k)}$ (B.4) associated with a skew invertible $\mathrm{R}$-matrix $R$ satisfy relations

$$
\operatorname{Tr}_{R}(i+1, \ldots, 2 i) \Upsilon_{R}^{(2 i)}=\left(\Upsilon_{R}^{(i)}\right)^{4}=\left(J_{1} J_{2} \ldots J_{i}\right)^{2}
$$

Proof. Calculation proceeds as follows:

$$
\begin{aligned}
& \operatorname{Tr}_{R}(i+1, \ldots, 2 i) \Upsilon_{R}^{(2 i)}=\operatorname{Tr}_{R}(i+1, \ldots, 2 i-1)\left(\Upsilon_{R}^{(2 i-1)}\left(\operatorname{Tr}_{R}(2 i) R_{2 i-1}\right)\left(R_{2 i-2} \ldots R_{1}\right)\right) \\
& =\operatorname{Tr}_{R}(i+1, \ldots, 2 i-1)\left(\left(R_{1} \ldots R_{i-1}\right) \Upsilon_{R}^{(2 i-1)}\left(R_{i-1} \ldots R_{1}\right)\right) \\
& \ldots=\left(R_{1} \ldots R_{i-1}\right)^{i} \Upsilon_{R}^{(i)}\left(R_{i-1} \ldots R_{1}\right)\left(R_{i-2} \ldots R_{1}\right) \ldots\left(R_{2} R_{1}\right) R_{1} \\
& =\left(J_{1} J_{2} \ldots J_{i}\right)\left(\Upsilon_{R}^{(i)}\right)^{2}=\left(\Upsilon_{R}^{(i)}\right)^{4} \text {. }
\end{aligned}
$$

Here in passing to the second line we calculated the R-trace $\operatorname{Tr}_{R}(2 i)$ with the help of (2.2.8) and then used (B.5) to move $(i-1) \mathrm{R}$-matrices to the left of the term $\Upsilon_{R}^{(2 i-1)}$. Expression in the third line results from similar calculations of the R-traces $\operatorname{Tr}_{R}(2 i-1), \ldots, \operatorname{Tr}_{R}(i+1)$, consecutively. Equalities in the last line result from rearranging factors of the product $\left(R_{1} \ldots R_{i-1}\right)^{i}$.

Acknowledgement. We are grateful to Ludwig Dmitrievich Faddeev for acquainting us with the problem of a dynamics of the isotropic q-top, and for numerous inspiring discussions and advice. We would like to thank Alexei Gorodentsev, Sergei Kuleshov, Andrey Levin, Dmitry Lebedev, Andrey Mudrov, Andrey Marshakov and Vyacheslav Spiridonov for their useful comments and conversations. We also would like to acknowledge the warm hospitality of the Max-Planck-Institute für Mathematik, where writing this paper was started in 2004 and finished in 2008. The work is supported by the Russian Foundation for Basic Research, grants No. 08-01-00392-a, and CNRS-RFBR No. 07-02-92166 and No. 09-01-93107.

\section{References}

[AF.91] Alekseev, A.Yu., Faddeev, L.D.: $\left(T^{*} G\right)_{t}$ : a toy model for conformal field theory. Commun. Math. Phys. 141(2), 413-422 (1991)

[AF.92] Alekseev, A.Yu., Faddeev, L.D.: An involution and dynamics for the $q$ deformed quantum top. Zap. Nauchn. Semin. LOMI 200, 3 (1992) (in Russian); English translation available at http:// arxiv.org/abs/hep-th/9406196, 1994 
[B] Burroughs, N.: Relating the approaches to quantized algebras and quantum groups. Commun. Math. Phys. 113, 91-117 (1990)

[ChP] Chari, V., Pressley, A.: A Guide to Quantum Groups. Cambridge: Cambridge University Press, 1994

[CS] Conway, J.H., Sloane, N.J.A.: Sphere Packings, Lattices and Groups. Berlin-Heidelberg-New York: Springer-Verlag, 1993

[D.86] Drinfeld, V.G.: Quantum Groups. In: Proceedings of the Intern. Congress of Mathematics, Vol. 1 (Berkeley, 1986), p. 798. For the expanded version see J. Math. Sci. 41(2), 898-915 (1988) (translated from Zap. Nauch. Sem. LOMI 155, 18-49) (1986)

[D.89] Drinfeld, V.G.: On almost cocommutative Hopf algebras. (Russian) Algebra i Analiz 1(2), 30-46 (1989); English translation in: Leningrad Math. J. 1(2), 321-342 (1990)

[DM.01] Donin, J., Mudrov, A.: $U_{q}(\mathrm{sl}(n))$-covariant quantization of symmetric coadjoint orbits via reflection equation algebra. Contemp. Math. 315, 61-79 (2002)

[DM.02] Donin, J., Mudrov, A.: Explicit equivariant quantization on coadjoint orbits of GL( $n, \mathbb{C})$. Lett. Math. Phys. 62(1), 17-32 (2002)

[ES] Etingof, P., Schiffmann, O.: Lectures on the dynamical Yang-Baxter equations. In: Quantum groups and Lie theory (Durham 1999), London Math. Soc. LN series 290, Cambridge: Cambridge Univ. Press 2001

[F.90] Faddeev, L.D.: On the exchange matrix for WZNW model. Commun. Math. Phys. 132(1), 131-138 (1990)

[F.94] Faddeev, L.D.: Current-like variables in massive nad massless integrable models. Lectures delivered at the International School of Physics 'Enrico Fermi'. Varenna, Italy, 1994; available at http://arxiv.org/abs/hep-th/9408041, 1994

[F.95] Faddeev, L.D.: Discrete Heisenberg-Weyl group and modular group. Lett. Math. Phys. 34(3), 249-254 (1995)

[F.99] Faddeev, L.D.: Modular double of a quantum group. In: Conf'erence Mosh'e Flato 1999, Quantization, Deformation, and Symmetries. Vol. I, Dordrecht: Kluwer Acad. Publ., 2000, pp. 149-156; available at http://arxiv.org/abs/math.QA/9912078, 1999

[FHIOPT] Furlan, P., Hadjiivanov, L.K., Isaev, A.P., Ogievetsky, O.V., Pyatov, P.N., Todorov, I.T.: Quantum matrix algebra for the $S U(n)$ WZNW model. J. Phys. A: Math. Gen. 36, 5497-5530 (2003)

[FRT] Faddeev, L.D., Reshetikhin, N.Yu., Takhtajan, L.A.: Quantization of Lie groups and Lie algebras. (Russian) Algebra i Analiz 1(1), 178-206; (1989) English translation in: Leningrad Math. J. 1(1), 193-225 (1990)

[GKL] Gerasimov, A., Kharchev, S., Lebedev, D.: Representation theory and quantum integrability. Progr. Math. 237, Basel: Birkhäuser, 2005, pp. 133-156, available at http://arxiv.org/abs/math. QA/0402112, 2004

[G] Gurevich, D.I.: Algebraic aspects of the quantum Yang-Baxter equation. (Russian) Algebra $\mathrm{i}$ Analiz 2, 119-148 (1990); English translation in: Leningrad Math. J. 2, 801-828 (1991)

[GPS.97] Gurevich, D.I., Pyatov, P.N., Saponov, P.A.: Hecke symmetries and characteristic relations on reflection equation algebras. Lett. Math. Phys. 41, 255-264 (1997)

[GPS.05] Gurevich, D.I., Pyatov, P.N., Saponov, P.A.: Cayley-Hamilton Theorem for Quantum Matrix Algebras of $G L(m \mid n)$ type. Algebra i Analiz 17(1) 160-182 (2005) (in Russian). English translation in: St. Petersburg Math. J. 17(1), 119-135 (2006)

[GPS.06] Gurevich, D.I., Pyatov, P.N., Saponov, P.A.: Quantum matrix algebras of the GL(m-n)type: the structure and spectral parameterization of the characteristic subalgebra. Teor. Matem. Fiz. 147(1), 14-46 (2006) (in Russian). English translation in: Theor. Math. Phys. 147(1), 460-485 (2006)

[GR.91] Gelfand, I.M., Retakh, V.S.: Determinants of matrices over noncommutative rings. Funct. Anal. Appl. 25, 91-102 (1991)

[GR.92] Gelfand, I.M., Retakh, V.S.: A theory of noncommutative determinants and characteristic funstions of graphs. Funct. Anal. Appl. 26, 1-20 (1992); Publ. LACIM, Montreal: UQAM, 14, pp. $1-26$

[GS.99] Gurevich, D., Saponov, P.: Quantum line bundles via cayley-hamilton identity. J. Phys. A: Math. Gen. 34(21), 4553-4569 (2001)

[GS.04] Gurevich, D., Saponov, P.: Geometry of non-commutative orbits related to Hecke symmetries. to appear in Contemp. Math.: Joseph Donin memorial volume, available at http://arxiv.org/abs/ math.QA/0411579, 2004

[H] Hlavaty, L.: Quantized braided groups. J. Math. Phys. 35, 2560-2569 (1994)

[HIOPT] Hadjiivanov, L.K., Isaev, A.P., Ogievetsky, O.V., Pyatov, P.N., Todorov, I.T.: Hecke algebraic properties of dynamical R-matrices: application to related quantum matrix algebras. J. Math. Phys. 40(1), 427-448 (1999) 
[I]

[IOP.98]

[KSch] Klimyk, A., Schmüdgen, K.: Quantum Groups and their Representations. Berlin: Springer, 1997

[M] Montgomery, S.: Hopf Algebras and their Actions on Rings. CBMS Lecture Notes Vol. 82, Providence, RI: Amer. Math. Soc., 1993

[Mum] Mumford, D.: Tata Lectures on Theta. I. Progress in Mathematics, Vol. 28, Boston, MA: Birkhäuser Boston Inc., 1983

[O] Ogievetsky, O.: Uses of quantum spaces. In: Proc. of School Quantum symmetries in theoretical physics and mathematics (Bariloche, 2000), Contemp. Math. 294, Providence, RI: Amer. Math. Soc., 2002 pp. 161-232

[OP.01] Ogievetsky, O., Pyatov, P.: Lecture on Hecke algebras. In: Proc. of the International School "Symmetries and Integrable Systems" (Dubna, Russia, June 8-11, 1999), JINR, Dubna, D2,52000-218, pp.39-88; MPIM Preprint 2001-40, available at http://www.mpim-bonn.mpg.de/ Research/MPIM-Preprint-Series/

[OP.05] Ogievetsky, O., Pyatov, P.: Orthogonal and symplectic quantum matrix algebras and CayleyHamilton theorem for them. Preprint MPIM2005-53; http://arxiv.org/abs/math.QA/0511618, 2005

[PP] Polishchuk, A., Positselski, L.: Quadratic Algebras. University Lecture Series, 37. Providence, RI: Amer. Math. Soc., 2005

[R.89] Reshetikhin, N.Yu.: Quasitriangular Hopf algebras and invariants of tangles. (Russian) Algebra i Analiz 1 (2), 169-188 (1989); English translation in: Leningrad Math. J. 1(2), 491-513 (1990)

[R.90] Reshetikhin, N.Yu.: Multiparameter quantum groups and twisted quasitriangular Hopf algebras. Lett. Math. Phys. 20, 331-335 (1990)

[RT] Reshetikhin, N.Yu., Turaev, V.G.: Ribbon graphs and their invariants derived from quantum groups. Commun. Math. Phys. 127(1), 1-26 (1990)

[S] Semenov-Tyan-Shanskii, M.A.: Poisson-Lie groups. The quantum duality principle and the twisted quantum double. (Russian) Teor. Mat. Fiz. 93(2) 302-329 (1992); English translation in: Theor. Math. Phys. 93(2), 1292-1307 (1992)

[SWZ.92] Schupp, P., Watts, P., Zumino, B.: Differential geometry on linear quantum groups. Lett. Math. Phys. 25(2), 139-147 (1992)

[SWZ.93] Schupp, P., Watts, P., Zumino, B.: Bicovariant quantum algebras and quantum lie algebras. Commun. Math. Phys. 157(2), 305-329 (1993)

[TW] Tuba, I., Wenzl, H.: On braided tensor categories of type bcd. J. Reine Angew. Math. 581, 31-69 (2005)

Communicated by L. Takhtajan 